\title{
QUAND LA RÉPUBLIQUE ARRIVE DANS LA MARINE DE GUERRE BRÉSILIENNE: QUEL PROJET «MODERNISATEUR» POUR LES ÉQUIPEMENTS ET POUR LE PERSONNEL? (1889-1910)
}

\author{
Silvia Capanema \\ Université Paris 13 - Pléiade EA 7338 \\ (France) \\ ORCID: https://orcid.org/0000-0002-1607-2055 \\ silvia.capanema@univ-paris13.fr
}

L'arrivée de la République au Brésil (1889) a permis, malgré de nombreuses continuités et le maintien de l'ordre social, la formulation de nouveaux projets pour mettre en place la «modernisation» des institutions nationales. La Marine de Guerre, dans un contexte d'importants changements technologiques et humains, devait aussi répondre aux attentes correspondant aux aspirations diplomatiques du pays d'expansion territoriale et de leadersbip en Amérique du sud, dans un contexte impérialiste. D'une part, cette «modernisation» a été construite à travers la mise en place des projets navals et l'acquisition d'importants bâtiments de guerre, modèles qui étaient de véritables références à leur époque. D'autre part, les différents ministres de l'Armada s'inquiétaient du manque d'effectifs pour les fonctions de marin subalterne et de la formation du personnel de base. Tout en gardant un ordre social inégal et hiérarchique, des écoles d'apprentis ont été pensées comme une réponse «moderne» dans ce contexte, pour l'époque. Les difficultés et contradictions internes se faisaient pourtant présentes à tous les niveaux, comme nous révèle une analyse attentive des sources diplomatiques et internes de l'Armada brésilienne (rapports, correspondances, publications, témoignages, photographies, livres de registres, etc.) pendant les deux premières décennies de la période républicaine. La révolte des marins de 1910 contre les châtiments corporels et pour de meilleures conditions de travail s'inscrit dans ce cadre modernisateur de la Marine, mettant en lumière aussi bien les contradictions que les efforts mis en place mais inachevés.

MoTS CLES: Marine brésilienne, Première République, modernisation, personnel, matériel. 


\section{WHEN REPUBLIC AROSE FOR BRAZIL'S IMPERIAL NAVY: THE PROSPECTS OF «MODERNIZA- TION» OF FLEET, EQUIPMENT, AND PERSONNEL FOR THE NAVY IN BRAZIL IN THE EARLY DECADES OF THE REPUBLIC}

Notwithstanding the numerous continuities, not least the preservation of the preceding social order virtually intact, régime change in Brazil, with the proclamation of the Republic (1889), made way for the conception and rethinking of a broad «modernizing» project for the country's institutions. The Navy, in a context of constant change (technological and buman), had also to answer to the country's diplomatic demands and aspirations, especially those related to territorial expansion and regional leadership, before a global landscape characterized by late nineteenth-century Imperialism. On the one hand, this «modernization» was carried out by means of an ambitious naval enterprise, with the purchase of large state-of-the-art war vessels. On the other hand, the various Navy ministers who succeeded one another were kept constantly busy with the insufficient number of conscripts and the poor training of men in the Navy's lower hierarchy. Although still tied to the same imbalanced and stratified social structure as ever, the many sailor schools sprouting across the country were conceived of as a «modern» answer to the Navy's concerns at the time. The obstacles and contradictions faced domestically were, nonetheless, striking, as a close reading of the contemporary diplomatic and the Navy's own internal documents (reports, correspondence, publications, books, and so on) dated from the two first decades of the republican period reveals. The Sailor's Revolt of 1910 against the institution of corporal punishment and for the implementation of better working conditions in the Navy can be understood as part of this «modernizing» intent, highlighting not only the contradictions, but also the nature of the efforts that were, in fact, taken, and yet much too limited in scope.

KEYWORDS: Brazilian navy, first republic, modernization, staff, equipments.

\section{Introduction}

A Rio de Janeiro, la nuit du 22 novembre 1910. Un groupe de plus de deux mille marins ordinaires de la Marine de guerre brésilienne se rebelle dans la baie de la Guanabara, prend le contrôle des principaux navires de la flotte, notamment les quatre grands cuirassés nommés Minas Gerais, São Paulo, Babia et Deodoro, et tourne ses armes contre le siège du pouvoir fédéral. Ces marins, en majorité des Noirs, des Métis et originaires du Nord et du Nord-est du pays demandaient en premier lieu la fin des châtiments corporels et plus particulièrement la suppression du fouet. Leurs autres revendications concernaient le remplacement des supérieurs «incompétents», l'augmentation des soldes, l'amélioration de la formation et l'instauration d'un nouveau barème de service. Dans les mots d'ordre, ils réclamaient «liberté» et «citoyenneté», alors que le Brésil avait définiti- 
vement aboli l'esclavage en mai 1888 - le dernier pays du continent — et que la République avait été instaurée en novembre $1889 .{ }^{1}$

Au Brésil comme dans le reste du monde occidental, le tournant des XIX et XXe siècles est marqué par une quête de modernisation poussée par les nouvelles découvertes dans le domaine des sciences et des technologies, impliquant le monde dans un nouveau cadre d'intégration et de production. L'expansion impérialiste de cette période, dans sa version coloniale comme dans sa version continentale poursuit un même but politique, ordonné par la notion de race. ${ }^{2}$ À la fin du XIX ${ }^{\mathrm{e}}$ siècle, le darwinisme social’ donne naissance aux théories eugénistes, tandis que la génétique s'attache à améliorer l'espèce humaine, en appliquant la notion de race à l'homme.

À l'aube du XX $\mathrm{X}^{\mathrm{e}}$ siècle, le projet impérialiste n'est pas étranger au Brésil. Après l'indépendance en 1822, un des piliers du projet brésilien est alors la conservation de l'unité nationale, qui compose un «Empire pour l'intérieur». Le pouvoir, concentré à Rio de Janeiro, exerce son emprise sur les provinces de l'intérieur du pays, en propageant un discours de civilisation, qui est également un discours de puissance. En ce sens, la quête de l'unité territoriale et politique est inséparable de la construction de l'identité brésilienne. Cet «Empire pour l'intérieur» est lié à la construction d'un peuple brésilien, sinon d'une «race brésilienne». ${ }^{4}$

Au Brésil, les présupposés du modèle libéral ont aussi aidé à penser autrement les institutions du pays: il serait donc nécessaire de faire face à l'esclavage et de moderniser le système politique. C'est pour cette raison que le décret d'abolition définitive de l'esclavage, signé au Brésil le 13 mai 1888 par la princesse régente, résulta autant de l'action des esclaves eux-mêmes, que des pressions exercées par divers secteurs sociaux à l'intérieur et à l'extérieur du pays, en accord avec les nouvelles valeurs du XIX siècle. ${ }^{5}$ Dans ce contexte politique, la Monarchie, qui isole le Brésil du reste des Républiques américaines, devient un symbole de retard. L'entrée du Brésil dans la nouvelle modernité politique comprend la mise en place d'une République pensée à la fois selon le modèle fédéraliste nord-américain et l'inspiration théorique française. La République semble d'autant plus appropriée que l'abolition de l'esclavage prive l'Empire de son principal point d'appui: les grands propriétaires ruraux. ${ }^{6}$

Les débuts de la République constituent pour les institutions officielles, telles que la

1. Cet article expose les principaux résultats de la première partie de ma thèse de doctorat dirigée par Juan Carlos Garavaglia et soutenue à l'EHESS, à Paris, en décembre 2009: Capanema P. de Almeida, Silvia, «'Nous, marins, citoyens brésiliens et républicains': identités, modernité et mémoire de la révolte des matelots de 1910», Thèse de doctorat en histoire, EHESS, Paris, décembre 2009.

2. Arendt, Hannah, Les origines du totalitarisme: l'impéralisme (1951), Paris, Fayard, 1982.

3. Schwarcz, Lilia Moritz, O espetaculo das raças: cientistas, instituições e questão racial no Brasil (18701930), São Paulo, Cia das Letras, 2005.

4. Mattos, Ilmar Rohloff de, «Construtores e herdeiros: a trama dos interesses na construção da unidade politica», Almanack Braziliense, n. 01, mai 2005.

5. Alonso, Angela, Flores, votos e balas: O movimento abolicionista brasileiro (1868-88), São Paulo, Companhia das Letras, 2015.

6. Costa, Emilia Viotti da, Da Monarquia à Republica: momentos decisivos, São Paulo, Grijalbo, 1977. 
Marine de guerre, un temps d'affirmation des idéaux de la modernité brésilienne. Toutefois, cela n'implique ni l'existence d'un seul projet uniforme ni que le projet de modernisation de la Marine soit un fait accompli. Au contraire, les idéaux républicains de modernité n'existant très souvent que dans les discours, souvent exprimés dans les rapports annuels élaborés par les différents ministres de la Marine (RMM).

\section{La «modernisation» des équipements}

La Marine brésilienne s'est consolidée pendant l'Empire (1822-1889). Elle fut un important acteur dans les guerres d'indépendance et dans celle de la province Cisplatine, ${ }^{7} \mathrm{du}-$ rant la période du premier règne de Dom Pedro I (1822-1831). Pendant la régence (18311940), l'Armada servit à la consolidation des frontières brésiliennes et à la répression des soulèvements provinciaux, devenant ainsi le garant de l'unité territoriale du Brésil. ${ }^{8}$ Sous le deuxième règne de Dom Pedro II (1848-1889), la Marine joua également un rôle important dans le cadre de la «consolidation de l'État National», s'impliquant dans la répression de la Traite Négrière à partir de 1850 et dans la Guerre du Paraguay. ${ }^{9}$

Contemporaine de la Révolution industrielle, la révolution des transports eut d'importantes conséquences sur la Marine brésilienne au XIX ${ }^{\mathrm{e}}$ siècle. Le passage du système de voile à la vapeur amena un changement dans la dynamique des voyages et du travail maritime, même si ce passage ne se produisit que progressivement. Les équipages étaient désormais moins nombreux, tandis qu'une nouvelle fonction apparaissait parmi les officiers (personnel d'encadrement) et les marins (personnel d'exécution): celle des machinistes, responsables de la production de l'énergie. Dépourvus de leurs voiles, les nouveaux vapeurs requéraient des timoniers, des hommes de veille et des rameurs pour le canot du bord. ${ }^{10}$

Dans un ouvrage de 1910, l'officier brésilien Arthur Dias estimait que le Brésil était la sixième puissance navale mondiale en ce qui concerne le nombre de bâtiments (cuirassés et vapeurs) en 1872, soit au lendemain de la Guerre du Paraguay. ${ }^{11}$ Cependant, la

7. Conflit impliquant l'Empire brésilien et les provinces Unies de La Plata, entre 1825 et 1828, pour la possession de la province Cisplatina, actuelle République Orientale de l'Uruguay, $c f$. Neves, Lucia Maria de Bastos das e Humberto Fernandes Machado, O Império do Brasil, Rio de Janeiro, Nova Fronteira, 1999.

8. Morel, Marco, A transformação dos espaços publicos: imprensa, atores politicos e sociabilidades na cidade imperial (1820-1840), São Paulo, Hucitec, 2005.

9. Voir: Arias Neto, José Miguel, Em busca da cidadania: praças da Armada nacional, 1867-1910; Thèse de doctorat, Departamento de Historia, Faculdade de Filosofia, Letras e Ciências Humanas, Universidade de São Paulo, 2001.

10. Lenhof, Jean-Louis, «Voile ou vapeur: le travail et la vie à bord des cargos français à la fin du XIX siècle (1880-1920)», Revue d'Histoire Maritime, n. 5, 2006, p. 80.

11. Les premières puissances étaient, dans l'ordre: l'Angleterre, Les États Unis, la France, la Russie, la Turquie, le Brésil, l'Espagne, L'Autriche, l'Allemagne, L'Italie, la Danemark, la Suède, la Norvège, le Portugal. Le Brésil était le seul pays sud-américain à figurer dans ce groupe selon cette source. Cf. Dias, Arthur, Nossa Marinha: notas sobre o renascimento da Marinha de guerra do Brazil no quatriennio de 1906 a 1910, Rio de Janeiro, Officinas Graphicas da Liga Maritima Brazileira, 1910, p. 45. 
Marine brésilienne aurait ensuite connu une phase de déclin durant les dernières années de l'Empire et les premières de la République. ${ }^{12}$

Un nouveau tournant vint enfin avec le projet républicain. La volonté de construire une République moderne, de devenir la puissance de l'Amérique du Sud eut aussi des conséquences sur la politique militaire et navale brésilienne, notamment quand le Baron de Rio Branco fut ministre des Affaires étrangères (1902-1912).

Le Baron de Rio Branco était un monarchiste libéral, ami de Dom Pedro II et fils du Vicomte de Rio Branco, sénateur et diplomate de l'Empire. ${ }^{13} \mathrm{Il}$ admirait les États-Unis et le fédéralisme, et méprisait les républiques latino-américaines pour leur désordre. À l'égard d'autres monarchistes, Rio Branco accepta le changement de régime, de peur que l'instabilité des révolutions latino-américaines ne se reproduise au Brésil. Tout en menant une politique étrangère adaptée aux intérêts des élites agro-exportatrices, ce diplomate sut mettre en œuvre une politique nationaliste ambitieuse, fondée sur l'ampleur du territoire brésilien, la croissance démographique et le potentiel de richesses naturelles du pays. ${ }^{14}$

La période Rio Branco marqua aussi un changement d'orientation de la politique extérieure brésilienne, lié à l'émergence de nouveaux partenaires économiques. Si la Grande-Bretagne fut le principal acteur et «allié» commercial du Brésil au XIX siècle, la politique étrangère brésilienne s'orienta vers les États-Unis à l'aube du Xx $\mathrm{X}^{\mathrm{e}}$ siècle, qui devint le principal marché d'exportation de la République brésilienne. La diplomatie brésilienne aurait donc accepté la doctrine Monroe et le corollaire Roosevelt et se serait positionnée en gardienne des intérêts nord-américains dans le sous-continent, afin de promouvoir ses propres ambitions. Rio Branco entendait ainsi maintenir de bonnes relations avec le principal importateur de café (plus de la moitié du café brésilien, principal produit du pays à l'époque, était exportée aux États-Unis) et garantir la distance militaire entre le continent américain et les puissances européennes dans un contexte fortement impérialiste. L'Itamaraty visait non seulement à la mise en place d'une «diplomatie agroexportatrice», mais aussi à l'expansion territoriale du Brésil (l'annexion du territoire correspondant à l'actuel État d'Acre, en Amazonie brésilienne, date de cette période) et, surtout, à la conquête du leadership sud-américain. ${ }^{15}$

Le développement des forces militaires, et notamment de la Marine, constituait un autre pilier de cette politique extérieure. L'expérience de la guerre russo-japonaise en 1904-1905 avait montré à tous les pays l'importance d'une Armada puissante pour le combat moderne. Ce conflit préfigure les guerres du XXe siècle, sur le plan militaire, tant

\section{Ibidem.}

13. De son vrai nom José Maria da Silva Paranhos Júnior (1845-1912). À la suite de son père, il reçut le titre de baron et le nom Rio Branco sous l'Empire. Diplomate, ministre, géographe et écrivain, il demeure le plus important symbole de la diplomatie brésilienne de nos jours. Voir: Moura, Cristina Patriota de, «Herança e metamorfose: a construção social de dois Rios Brancos», Estudos Historicos, n. 25, 2000/1, pp. 81-101.

14. Bueno, Clodoaldo, Politica externa da Primeira Republica: os anos de apogeu - de 1902 a 1908, São Paulo, Paz e Terra, 2003, pp. 127-144.

15. Ibidem. 
par sa durée ( 1 an et demi) et l'ampleur des forces engagées (plus de 2 millions d'hommes) que par l'emploi des techniques les plus modernes de l'art de la guerre (logistique, lignes de communication et de renseignements, combinaison entre les opérations terrestres et maritimes, etc). ${ }^{16}$ Les rivalités avec la République Argentine, nourries avec force des deux côtés de la frontière, fournissaient un argument de plus aux investissements dans le matériel de guerre. Enfin, la Marine nationale contribuait à assurer la présence du drapeau brésilien dans plusieurs pays, à travers ses missions et déplacements dans le monde. C'est dans ce cadre que s'inscrit le besoin d'élaborer un véritable projet naval pour la Marine de guerre brésilienne, fait inédit à l'époque.

Déjà de 1893 à 1902, les différents Rapports des Ministres de la Marine —désormais $R M M$ dans le texte- ${ }^{17}$ signalèrent le besoin d'acquérir de nouveaux équipements. Dans le rapport sur l'année 1893, en raison des pertes de la dite «Révolte de l'Armada» —une révolte d'officiers en 1893 suite à l'instabilité du début de la République-, le ministre évoquait déjà la nécessité de réparer le matériel existant et d'augmenter la force navale brésilienne grâce à l'acquisition de deux scouts de première classe et de six chasse-torpilleurs. L'année suivante, le ministre Elisario J. Barbosa ajoutait à cette requête une demande d'acquisition de deux cuirassés de petites dimensions. Il lançait ainsi les bases d'un débat promis à un bel avenir: le choix nécessaire entre les grands cuirassés et les petits bâtiments (croiseurs rapides, les canonnières, les torpilleurs et le sous-marin).

Ce débat n'était pas limité à la Marine brésilienne. En France, le choix des petits bâtiments de guerre rapides et manoeuvrants face aux grands cuirassés fut au centre du projet de la Jeune École dans la seconde moitié du XIX siècle. La construction des premiers cuirassés à partir des années 1860 encouragea la construction de grands bâtiments très coûteux partout dans le monde, mais l'apparition de la torpille quelques années plus tard inversa cette tendance, fournissant des arguments à ceux qui soutenaient le choix des petites unités. Le contre-amiral Aube développa, à partir des années 1875, une théorie selon laquelle, dans le combat naval, il y avait une tendance à la neutralisation des pouvoirs des cuirassés, les conflits se limitant donc à la guerre de course contre l'ennemi. Quoique cette politique obtînt de bons résultats dans les interventions d'outre-mer, les croiseurs étant les bâtiments les plus adéquats dans ce contexte, elle n'eut pas que des conséquences positives sur la Marine française: la France fut dotée de nombreuses petites unités très coûteuses et la Jeune École fut jugée responsable du retard naval du pays à la veille de la guerre de 1914-1918 (par rapport à l'Angleterre et à l'Allemagne). De fait, le modèle défendu par la Jeune École était plus adapté à la défense qu'à l'attaque. ${ }^{18}$

En contrepoint de ce modèle, l'amiral nord-américain Alfred Thayer Mahan écrivit une série d'études sur les combats maritimes dans la seconde moitié du XIX ${ }^{\mathrm{e}}$ siècle. Selon

16. Duby, Georges (dir.), Grand atlas historique, Paris, éditions Larousse, 2006.

17. Ces rapports des ministères brésiliens sont disponibles sur le portail du Center of Researches Libaries: $<$ http://www.crl.edu/brazil $>$.

18. Verge-Franceschi, Michel, Dictionnaire d'histoire maritime. Paris: Editions Robert Laffont, 2002, pp. 801-802. 
lui, le combat moderne était destiné à être mener en haute mer et non le long des côtes, dans une stratégie plutôt offensive que défensive. En conséquence, il réfutait le rôle des courses et soulignait le rôle des grands bâtiments de guerre pour les grandes puissances. Il fut lui aussi critiqué au lendemain de la Grande Guerre, pour n’avoir pas prévu l'importance croissante des sous-marins dans les conflits modernes. ${ }^{19}$

Inspiré par ce débat international, le sénateur, écrivain et juriste Rui Barbosa écrivit un article intitulé «Lição do Extremo Oriente» (Leçon de l'Extrême Orient) lors de son exil en Angleterre. ${ }^{20}$ À partir de l'exemple du conflit sino-japonais, il affirmait que le Brésil devait se doter d'une flotte navale puissante pour se prémunir contre la guerre (plus que pour la provoquer), garantir son hégémonie et l'équilibre entre les pays. À cette époque, moderniser la Marine brésilienne signifiait rentrer dans la course aux armements et suivre les tendances des pays du Nord. Ainsi, selon le RMM de 1896, 8 navires brésiliens étaient déjà commandés, même si l'on ne pouvait pas espérer de délais de construction très courts, «puisque les finances du pays ne le permettaient pas». Une certitude demeurait: les commandes devaient être faites aux chantiers européens.

Le Brésil, cherchant à combler ces besoins et à s'inscrire dans les débats de son époque, avait du mal à se rendre compte aussi bien de la publicité existant derrière ces affaires que de la plongée du pays dans un dans cycle de dépendance des nations considérées plus avancées. Sans doute à cause de ces coûts élevés et du mauvais état des finances pendant la période de la présidence de Prudente de Morais, le RMM de 1897 évoque les difficultés rencontrées par le gouvernement brésilien pour régler les commandes de navires passées en Europe, ainsi que le manque de soldats et de marins.

Au cours des années qui suivirent, la réparation des navires de la flotte existante et l'«acquisition de personnel et de matériel» apparurent tour à tour comme «le problème le plus inquiétant» pour la Marine. ${ }^{21}$ Ces besoins étaient, dans une large mesure, justifiés par le contexte international, marqué par les conflits entre le Japon et la Chine (18941895 ) et entre les États-Unis et l'Espagne (1898). Le Brésil, quant à lui, disputait le leadership sud-américain à l'Argentine et au Chili, comme l'observa le ministre Carlos Ballbazar da Silveira dans son rapport de 1898: «J'ai la ferme conviction qu'il est nécessaire et indispensable que nous ayons une force navale qui nous garantisse, sinon la suprématie dans l'Amérique du Sud telle que nous l'avons déjà connue, du moins une position noninférieure aux autres Marines de cette partie du continent». ${ }^{22}$

De fait, le Brésil n'était pas le seul pays du sous-continent à s'inquiéter du devenir de sa flotte. En 1900, l'ambassadeur brésilien à Buenos Aires écrivit une lettre à son ministre de tutelle afin de l'avertir du considérable développement la Marine de guerre argentine, qui était passée de 6.114 tonnes en 1875 à 94.891 tonnes en 1900. Il en concluait que

19. Ibidem, p. 900.

20. Barbosa, Rui, «Lição do Extremo Oriente», In: Cartas da Inglaterra [1895], São Paulo, Iracema, 1972, pp. 11-92.

21. $R M M, 1899$, p. 48.

22. $R M M, 1898$, p. 32. 
l'Argentine jouissait désormais d'une supériorité d'armements et que le Brésil n'était plus la première puissance navale du sous-continent, place qu'il convenait de récupérer à tout prix. $^{23}$

Au Brésil, le projet naval gagna finalement une première formulation en 1903, après le rétablissement de l'équilibre des comptes sous la présidence Campos Salles et l'investiture de Rodrigues Alves, qui affichait une volonté de «modernisation». Dans son discours d'investiture, celui-ci avait annoncé qu'il souhaitait accorder une attention spéciale aux forces armées, de terre et de mer et «promouvoir des améliorations compatibles». ${ }^{24}$ Le nouveau ministre Júlio César de Noronha en profita pour demander au gouvernement des crédits pour la réalisation d'un projet sur six à huit ans. Dans son rapport de 1903 , le ministre présentait une synthèse de son projet naval, désigné depuis par les appellations «projet de 1904» ou «projet de Júlio César de Noronha»:

Voici donc, en résumé, mon programme, qui doit se réaliser dans les six à huit ans à venir: 3 cuirassés de 12.500 à 13.000 tonnes de déplacement; 3 croiseurs cuirassés de 9.200 à 9.700 tonnes; 6 chasse-torpilleurs de 400 tonnes; 6 torpilleurs de 130 tonnes; 6 torpilleurs de 50 tonnes; 3 sous-marins; 1 vapeur charbonnier capable de porter 6.000 tonnes de carburant. ${ }^{25}$

Dans son modèle proportionnel 3 par 3, ce plan était probablement inspiré de la Marine japonaise après la bataille de Port-Arthur (une des plus sanglantes de la guerre russo-japonaise), qui lui permettait de souligner le pouvoir des torpilleurs et des destroyers, les contre-torpilleurs. Il fut le premier véritable projet naval brésilien intégré, les commandes réalisées auparavant ne concernant que des bâtiments individuels. Lors de sa présentation au Congrès, les députés en discutèrent les finalités —la compétition avec l'Argentine et le leadership sud-américain- et s'accordèrent sur la nécessité d'investir également dans la formation du personnel. ${ }^{26}$

Le rapport de l'année suivante nous informe que le Congrès approuva le projet à une quasi-unanimité et le convertit en loi par le décret 1296 du 14 décembre 1904. ${ }^{27}$ Le rapport de 1905 fut le dernier signé par le vice-amiral Júlio Cesar de Noronha. Il informait que, après une étude comparative menée auprès de différentes compagnies étrangères, le gouvernement avait décidé de confier la construction des cuirassés de 13.000 tonnes aux chantiers Armstrong Whitworth \& Co. Limited, qui «proposait le meilleur prix, parmi tous ceux répondant aux conditions exigées». ${ }^{28}$

Toutefois, le choix de la société anglaise semble avoir été motivé par d'autres raisons. Pour répondre à l'offre brésilienne, les chantiers Armstrong avaient travaillé avec Vic-

23. Bueno, Clodoaldo, Politica externa da..., cit., pp. 288-232.

24. Arias Neto, José Miguel, Em busca da..., cit., p. 234.

25. RMM, 1903, p. 11. Les traductions en français ont été faites par l'auteure de l'article.

26. Martins Filho, João Roberto, A Marinha brasileira na era dos encouraçados, 1885-1910, Rio de Janeiro, editora FGV, 2010.

27. RMM, 1904, p. 4.

28. $R M M, 1905$, p. 5 
kers, Sons \& Maxim, le deuxième leader britannique de la construction navale, pour le dessin des navires. Selon des documents du Foreign Office britannique datés de mi-octobre 1906, des «intérêts personnels privés jouèrent un important rôle dans la commande des navires, qui n'aurait jamais réussi sans les gros pots de vin distribués aux plus hautes autorités navales et à d'autres personnages impliqués». ${ }^{29}$ Ainsi, il fut prévu que Armstrong Whitworth \& Co. serait chargé de la construction de deux cuirassés, baptisés par les Brésiliens Minas Gerais et Rio de Janeiro, tandis que Vickers s'occuperait de la construction du troisième, le São Paulo, la durée de la construction étant estimée à environ deux ans.

Bien qu'il eût défendu ardemment son projet, il fut remplacé par Alexandrino de Alencar en 1906 en raison de l'obsolescence de son projet à l'échelle internationale. Né dans le Rio Grande do Sul en 1848 de père officier dans l'Armée de Terre, le nouveau ministre était lui-même un ancien officier de la Guerre du Paraguay et un rebelle de 1893. Exilé un temps après la Révolte de l'Armada, il fut amnistié et revint au Brésil où il occupa le poste de ministre de la Marine entre 1906 et 1910 sous la présidence d'Afonso Pena, puis entre 1913 et 1918 et entre 1922 et $1926 .{ }^{30}$

Tandis que le Brésil gardait toujours ses anciens bateaux, le lancement du dreadnought par la force royale britannique eut un fort impact dans les quatre parties du monde. L'engouement pour ce nouveau bâtiment profita aux chantiers anglais qui proposèrent aux autorités brésiliennes de remplacer les cuirassés déjà commandés par des modèles de dreadnoughts en 1906.

\section{Le projet naval de 1906: Alexandrino de Alencar et la «course vers la mer»}

Peu après son entrée en fonction, Alexandrino de Alencar rejeta le projet de Noronha et jugea les cuirassés de 13.000 tonnes, qui avaient été commandés par «malheur» ou par «manque de chance», «en dessous de toute critique». ${ }^{31} \grave{A}$ ses yeux, une nouvelle ère s'ouvrait, marquée par la disparition des armements moyens.

En conséquence, les chantiers anglais commencèrent à démanteler les constructions en cours et à dessiner de nouveaux équipements. Le 20 février 1907, un nouveau contrat fut signé, qui ne prévoyait que deux bâtiments, le Minas Gerais et le São Paulo, à livrer dans les plus brefs délais. ${ }^{32}$ La construction du Minas Gerais débuta le 17 avril 1907 à Elswick, tandis que le São Paulo vit le jour à Barrow le 24 septembre de la même année.

Il apparaît donc que le projet de 1906 remplaçait les deux cuirassés de 13.000 tonnes

29. Barclay a Bart, Petrópolis, October 15, 1906. FO 371/13, 37222, p. 251. Cité par Martins Filho, João Roberto, A Marinha brasileira..., cit.

30. Alexandrino, «le Grand Amiral», a le statut d'un héros dans la mémoire de la Marine. Il fut aussi sénateur en 1906 et entre 1921 et 1922. Voir: ALENCAR, Carlos Ramos de, Alexandrino, o grande marinheiro, Rio de Janeiro, Serviço de Documentação Geral da Marinha, 1989.

31. Martins Filho, João Roberto, A Marinha brasileira..., cit., p. 29.

32. Ibidem, p. 35 
(réévalués par la suite à 15.000 tonnes) par deux cuirassés modèle dreadnought de 18.000 tonnes. Quant au troisième, le Rio de Janeiro, il ne rejoignit jamais la Marine brésilienne, mais incorpora la flotte de Turquie, où il servit pendant la Première Guerre Mondiale. L'historiographie avance deux facteurs majeurs pour expliquer cette annulation: la pression des autres puissances sud-américaines (notamment de l'Argentine) et la question économique.

Les débats sur la modernisation de la Marine soulevèrent, côté argentin, des arguments propres à une véritable course aux armements. ${ }^{33}$ Le plan naval brésilien servit ainsi de justification au projet argentin d'achat de deux cuirassés modèle dreadnought. Pour cela, l'Argentine devait renoncer au pacte d'équivalence navale signé avec le Chili en 1902, selon lequel les deux pays s'engageaient à ne pas acheter de nouveaux équipements. ${ }^{34}$ Ainsi, le périodique La Prensa avait porté son attention sur la politique de Rio Branco, qui visait à faire du Brésil le leader du sous-continent, y compris dans le domaine militaire. Le gouvernement argentin, selon l'opinion de ce journal, ne pouvait accepter une position d'infériorité face au Brésil et devait donc élaborer un projet naval au moins équivalent à celui de son voisin. ${ }^{35}$ La Nación, cependant, prônait une position différente, remarquant que le plan naval brésilien était très coûteux et que d'autres dépenses étaient plus judicieuses pour assurer le progrès de l'Argentine, du Brésil et du Chili. ${ }^{36}$

Rio Branco, toutefois, n'était pas le mentor du projet de 1906. Certes, il était favorable à la construction d'une Armée et d'une Marine puissantes, mais son choix se portait plutôt sur l'acquisition de plusieurs petits cuirassés que sur l'achat de deux ou trois grands bâtiments, dans la lignée du programme Júlio de Noronha. ${ }^{37}$ Mais, si Rio Branco n'avait été ni un stratège ni un défenseur convaincu du projet de 1906, les achats d'armements confortèrent toutefois ses positions en terme de politique extérieure, ce dont témoigne la création d'une légation militaire à l'ambassade aux États-Unis et la participation du Brésil aux conférences de La Haye en 1899 et 1907.

Ces commandes des grands bâtiments parurent étranges aux diplomates français en poste au Brésil, comme en témoigne cette note de 1908:

Les chantiers anglais lançaient il y a quelques jours le cuirassé Minas-Gerais, du type dreadnought, destiné à la Marine du Brésil et qui est aujourd'hui le plus puissant navire de guerre à flot. Deux autres cuirassés du même type, le Rio de Janeiro et le São-Paulo [qui] sont en construction en Angleterre, ainsi qu'une certaine quantité de scouts, de destroyers, de contre-torpilleurs, de sous-marins, qui doivent faire du Brésil la neuvième puissance navale du monde.

33. Bueno, Clodoaldo, Politica externa da..., cit.

34. Heinsfeld, Adelar, «Estanislau Severo Zeballos: artifice da politica armamentista argentina no inicio do século XX», communication présentée dans la 26a. Réunion de la Sociedade Brasileira de Pesquisa Historica, julho 2006.

35. La Prensa, 20.10.1908. Cité par Heinsfeld, Adelar, «Estanislau Severo...», cit.

36. Les périodiques El Pais et El Diario ont défendu une opinion également plus modérée: les dépenses provoquées par ces bâtiments étaient trop importantes et l'Argentine devait avoir d'autres priorités. Voir: Bueno, Clodoaldo, Politica externa da..., cit., pp. 195-219.

37. Ibidem, p. 223. 
(...) À quel but immédiat répondent ces armements? S'agit-il d'un accès, coûteux d'ailleurs, de mégalomanie? Ou bien le Brésil redoute-t-il réellement un conflit avec la République Argentine, au sujet de la juridiction des eaux du Rio de la Plata? ${ }^{38}$

Ces succès dans les affaires maritimes furent utilisés pour appuyer la demande d'un attaché militaire français au Brésil. Plusieurs lettres furent échangées en ce sens, qui développaient autant des arguments positifs (amélioration du partenariat franco-brésilien en matière militaire) que négatifs (rivalité avec l'Angleterre et surtout l'Allemagne) pour justifier cette requête:

L'Allemagne, il est vrai, se contente, pour l'instant, d'un simple officier attaché à sa Légation, mais l'Angleterre, les États-Unis et l'Argentine ont des attachés navals ou militaires, suivant le cas. Cet acte de courtoisie ne pourrait que flatter les Brésiliens et comme on peut, sans crainte de se tromper, prévoir qu'il sera, tôt ou tard, accompli par l'Allemagne mieux vaudrait à mon avis, le précéder que la suivre. ${ }^{39}$

\section{Vive le «Minas Gerais», le géant de la mer}

Le programme du ministre Alencar, qu'il avait lui-même surnommé Rumo ao Mar («course vers la mer»), se réduisit finalement à deux dreadnoughts, deux scouts et dix destroyers délivrés par des chantiers internationaux au Brésil à partir de 1908.

Incorporé en premier et un petit peu plus puissant que le São Paulo, le Minas Gerais fut le bâtiment le plus fêté et le plus commenté au Brésil. Son arrivée le 17 avril 1910 fut célébrée avec enthousiasme par la population brésilienne, comme en témoigna le quotidien $O$ Paiz dès le lendemain:

L'arrivée du Minas Gerais, voici le grand événement qui a fait hier vibrer la corde patriotique de l'âme nationale tout entière, car ce n'est pas que Rio de Janeiro qui a reçu dans les eaux de sa magnifique baie le formidable dreadnought... ça a été le Brésil entier qui a salué, par les géants contours du colosse des mers sud-américaines, le symbole souverain de sa propre richesse, la concrète expression de son énergie en tant que nation (...) le peuple brésilien (...) portant des milliers des mouchoirs ou criant des «vives» chaleureux, a manifesté son immense joie. (...) Les Brésiliens saluaient, par les contours en acier du Minas Gerais, le Brésil nouveau, opulent et puissant empruntant le chemin du progrès et de la civilisation avec autant de gaillardise que le premier de ses dreadnoughts — le premier dreadnought au monde- fendant les eaux de la baie de Guanabara. ${ }^{40}$

38. Archives du Quai d'Orsay, Correspondance politique et commerciale, Défense Nationale, vol. 3, 24/09/1908.

39. Ibidem.

40. O Paiz, 18/04/1910. 
Cet extrait montre clairement que le Minas Gerais était un symbole autant qu'un navire de guerre: il signifiait le pouvoir, la puissance, la beauté, le progrès et la civilisation. Incarnation de la modernisation technique souhaitée par la Marine du début du XXe siècle, ce grand dreadnought fut aussi chanté par un comédien et compositeur populaire de l'époque: Eduardo das Neves, un clown, noir, soldat dans les sapeurs pompiers, d'origine modeste, qui animait les rues de Rio avec ses chansons et ses performances. Il enregistra plusieurs chansons entre 1895 et 1919, dont Minas Gerais écrite sur la mélodie napolitaine Vieni sul mare, qui devint par la suite l'hymne de l'État du Minas Gerais sous le titre Oh, Minas Gerais! (avec des paroles modifiées dans deux versions différentes). Eduardo das Neves, surnommé Dudu das Neves, s'inspirait très souvent des événements quotidiens et des tensions sociales et raciales pour écrire ses chansons. ${ }^{41}$ En témoignent les paroles de Minas Gerais:

Lauriers du Triomphe / Le siècle nous apporte / Allons saluer le géant de la mer/ Oh, Minas Gerais/Vive l'Armada, virile, brésilienne/ Qui aujourd'hui peut chanter fière/ Elle est dans la mer du Sud la première/ Car elle exhibe le géant de la mer/ L’Armada ne craint plus les pouvoirs navals/ Elle est aussi puissante et virile/ Il suffit de la force du Minas Gerais/ Pour la défense du Brésil.

Même si l'Angleterre, la France et l'Allemagne allaient construire d'autres navires du modèle dreadnought avant 1914 (pour la Première Guerre Mondiale, l'Angleterre en alignait 31, l'Allemagne 21, la Russie 7, la France, l'Italie et l'Autriche 4), ces grands bâtiments brésiliens, le Minas et le São Paulo, furent très vite dépassés par la nouvelle course d'armements provoquée par la Grande Guerre. ${ }^{42}$ Et le Brésil ne fut pas en mesure de suivre cette concurrence.

Juste après la révolte des marins en novembre 1910 contre les châtiments corporels, plusieurs articles et caricatures dans la presse furent aussi très critiques quant à l'utilité de ces bâtiments de guerre; ils étaient comparés à de véritables «éléphants blancs». ${ }^{43}$

\section{Le projet de «modernisation» pour le personnel}

Tout au long du XIXe siècle, la consolidation des cadres fixes, aussi bien pour les marins que pour les officiers, est une préoccupation constante pour le gouvernement brésilien. Toutefois, le recrutement forcé constituait à cette époque la modalité la plus fréquente d'enrôlement, ${ }^{44}$ avec pour résultat des origines sociales très similaires des marins et sol-

41. Abreu, Martha. (2010). O «crioulo Dudu»: participação política e identidade negra nas histórias de um músico cantor (1890-1920). Topoi (Rio de Janeiro), 11 (20), pp. 92-113.

42. Taillemite, Etienne, L'Histoire ignorée de la Marine française, Paris, Perrin, 1999, pp. 389-395.

43. Capanema P. de Almeida, Silvia, «Nous, marins, ...», cit.

44. Greenhalgh, Juvenal, $O$ arsenal de Marinha do Rio de Janeiro na bistória: 1822-1889, Rio de Janeiro, IBGE, 1965, p. 57. 
dats de l'armée de guerre: exclus, expropriés, hommes libres pauvres et esclaves. Très souvent, le recrutement se faisait par le biais de la police, qui envoyait des condamnés à l'Armée de Terre et à la Marine de guerre, ainsi que des marins de la Marine marchande capturés de force sur les navires sur lesquels ils servaient. ${ }^{45} \mathrm{La}$ création de compagnies fixes donna naissance au Corpo de Imperiais Marinheiros (Corps des Marins Impériaux) en 1840, auquel furent jointes des compagnies d'apprentis marins, destinées aux mineurs âgés de 10 à 17 ans. Le recrutement forcé, qui constituait la modalité d'incorporation la plus fréquente des soldats dans la Marine de guerre jusqu'alors commença à perdre du terrain au profit des compagnies d'apprentis marins à partir de la moitié des années 1870 . En effet, ces dernières connurent une croissance presque constante à partir de l'année 1859.

Plusieurs rapports de ministres attestent la volonté de mettre fin à la pratique du recrutement forcé. Répondant à ces aspirations, une nouvelle loi fut approuvée en 1874 pour le recrutement dans l'Armée de Terre et dans la Marine. Les soldats devaient désormais être recrutés parmi les volontaires, les engagés et les réengagés ou, au besoin, par tirage au sort. Ce tirage au sort excluait néanmoins plusieurs catégories d'individus: les diplômés, les séminaristes, les ecclésiastiques, les personnes ayant une famille à charge, les policiers, les marins marchands, les propriétaires ou administrateurs d'usines ou de fermes de plus de dix employés, les cheminots, les machinistes des embarcations à vapeur, les domestiques des fermes de bétail, etc. Ces exceptions visaient à préserver, d'une part, le marché du travail — qui devait faire face à un déficit de main-d'œuvre suite à l'interdiction de la traite négrière- et, d'autre part, les fils des familles les plus aisées. En fin de compte, la Marine continuait à recruter parmi les hommes jeunes de préférence célibataires, libres, pauvres et sans occupation. La loi du tirage au sort n'eut pas de résultats concrets ni à l'époque ni sous la République.

Après l'abolition de l'esclavage en 1888, la Marine de guerre souffrit de plein fouet la concurrence de la Marine marchande en matière de recrutement. En effet, le service militaire risquait de ne plus fournir les forces suffisantes à partir du moment où le travail asservi -auquel les longues années du service obligatoire pouvaient présenter quelque ressemblance- devenait anachronique. En outre, le manque chronique de bras fut aggravé par le nombre croissant de désertions à la fin de l'Empire.

\section{Des marins et soldats pour la République}

L'instauration de la République brésilienne en 1889 coïncida, avec une année d'écart, non seulement avec la fin officielle de l'esclavage, mais aussi avec un moment de découvertes scientifiques et techniques qui provoquèrent des changements dans la composition du personnel de la Marine de guerre. Un mois et demi après la proclamation de la Répu-

45. Beattie, Peter, The Tribute of Blood: Army, Race and Nation in Brazil, 1864-1945, Durham, Duke University Press, 2001. 
blique, le corps d'officiers de l'Armada fut réformé introduisant l'expression «officier combattant» pour différencier les membres du Corps de l'Armada des ingénieurs et des médecins. ${ }^{46}$ Ces différentes mesures augmentèrent la proportion d'officiers dans les échelons les plus élevés et diminuèrent le nombre d'officiers intermédiaires entre les hauts gradés et les marins, augmentant l'écart entre les corps d'officiers et celui des marins.

Outre la recomposition hiérarchique de 1889, la complexification technique et le besoin croissant d'armements plus puissants furent à l'origine de la création du Corps d'Ingénieurs Navals (décret 327 du 12/04/1890) dont les fonctions étaient clairement distinctes de celles des officiers de bord ${ }^{47}$ L'accès à ce Corps d'Ingénieurs se faisait par un concours, dont l'inscription était réservée aux sous-lieutenants ayant reçu le diplôme de l'École Navale avec mention. Ceux qui obtenaient les meilleures places au concours partaient en Europe ou aux États-Unis pour étudier la spécialité de leur choix. Ces changements sont traditionnellement interprétés comme:

Une conséquence, sans doute, du programme naval du début du siècle (1906-1910), qui donna au Brésil une Marine de guerre appréciable et modernisée. (...) Le navire de guerre, plus détaillé, constituait dorénavant, par la multiplicité de sa mécanique, un ensemble si complexe et dispendieux qu'on ne pouvait plus le confier à des professionnels sans formation spécifique. ${ }^{48}$

Quant aux marins subalternes, ils faisaient partie d'un autre corps du personnel de la Marine. Le recrutement étant toujours prévu selon les termes de la loi 2556 du 26/09/1874: 1) les mousses originaires des écoles d'apprentis; 2) l'engagement et le réengagement des volontaires; 3 ) en cas de manque de volontaires, par le tirage au sort des citoyens brésiliens se trouvant sur les listes. Le Corps de Marins Impériaux devint Corps des Marins Nationaux et son effectif fut fixé à 4.000 hommes en état complet, soit une augmentation de 700 marins par rapport au total estimé de l'année $1888 .{ }^{49} \mathrm{La}$ tendance au sous-effectif et les problèmes de recrutement continuèrent cependant.

Afin de surmonter ces difficultés, on procéda à des changements dans la législation et dans la structure de la Marine avec notamment: l'établissement d'un nouveau Code Pénal (décret 18 du 07/03/1891), l'abolition des châtiments corporels (prévue dans la Constitution de 1889), l'augmentation des soldes des Marins Nationaux et du Bataillon Naval (décret $74 \mathrm{~A}$ du 20 décembre 1889) et la réduction du service obligatoire à 9 ans. Ces changements, destinés à attirer un plus grand nombre de volontaires, ne furent toutefois pas mis en pratique dans leur intégralité et, selon les rapports des ministres établis entre 1889 et 1908, eurent très peu d'impact sur le recrutement. Les châtiments corporels, par

46. Caminha, Herick Marques, Historia administrativa do Brasil: organização administrativa do Ministério da Marinha na Republica, Brasilia/Rio de Janeiro, Serviço de Documentação da Marinha, 1989, pp. 126-159.

47. RMM, 1890, p. 8.

48. Scarvada, Levy, História do corpo de suboficiais da Armada, Imprensa Naval, Rio de Janeiro, 1954. Cité par Caminha, Herick Marques, Historia administrativa..., cit., p. 124.

49. RMM, 1890. 
exemple, furent abolis par le deuxième décret de la République, mais rétablis juste après suite aux pressions des officiers de bord pour garder la discipline à bord. ${ }^{50}$

Être soldat dans la Marine n'éveillait pas un grand intérêt de la population en général, étant donné les faibles soldes et les dures conditions de travail. Et pour cause, la solde des mousses (grumetes) ou apprentis représentait seulement $1 \%$ du revenu des amiraux, tandis que celle des marins ne dépassait pas $10 \%$ de cette valeur. De fait, les premières années du nouveau régime furent caractérisées par un sous-effectif important dans la Marine brésilienne, notamment entre 1893 — l'année de la révolte des officiers_ et 1902; et en 1911 —juste après la révolte des matelots contre les châtiments corporels.

Tous les ministres de la période étudiée s’inquiétaient du mépris dont les Brésiliens entouraient les marins. Dès 1890, le ministre Eduardo Wandenkol évoquait ce sujet:

Malgré l'abolition des châtiments corporels et les avantages offerts par le décret 673 du 20 août 1890 et par plusieurs circulaires de mon prédécesseur concernant les individus désireux de s'engager, nous ne sommes pas parvenus à compléter [le Corps des Marins Nationaux], ni même à obtenir le nombre de soldats strictement nécessaire pour remplir les vides produits par les désertions et les pertes par décès, incapacité physique ou conclusion du temps de service. (...) L'abolition du recrutement forcé et la suppression, selon les termes des articles 3 et 4 de la Constitution, des primes pour les engagés compliqueront encore l'acquisition de personnel pour le service, tant que ne sera pas organisé le tirage au sort prévu dans l'article 4, si grande est la répugnance pour la vie maritime militaire dans notre population». ${ }^{51}$

Durant presque toute la période, les désertions provoquèrent les pertes les plus importantes, suivies par les licenciements — pour fin de contrat ou incapacité physique_ et les décès —dus le plus souvent à des maladies, quelques fois à des «désastres» maritimes. Pendant la période étudiée, les réformes (retraites) étaient très rares dans le Corps de Marin Nationaux, la plupart de ses membres ne parvenant pas à compléter les années nécessaires pour devenir pensionnaires de l'Armada brésilienne.

\section{La face cachée du recrutement forcé}

Le nouveau Code Pénal de la République, daté de 1890, ouvrait des dispositifs qui permettaient la communication entre la répression policière et les forces armées. Il établissait des contraventions pour «vagabondage, mendicité ou capoeiragem» (les articles 399 à 404), qui visaient directement les populations pauvres.

Le terme capoeiragem désignait alors la pratique de la capoeira, une technique de combat apportée et développée par les esclaves au Brésil, mais c'était aussi une forme de résistance et d'intimidation que les personnes issues des couches populaires utilisaient en

50. Capanema P. de Almeida, Silvia, «Nous, marins, ...», cit.

51. RMM, 1890, p. 13. 
cas de conflits (internes ou contre la police). Le «capoeira» désignait un homme connaissant cette culture, maîtrisant les coups de cette lutte et ayant parfois recours à d'autres armes comme le couteau ou une canne. ${ }^{52}$

Entièrement consacré aux «vagabonds et capoeiras», l'article 13 du Code pénal de 1890 stipule que toute personne dépourvue d'occupation, de ressources ou de garant, risquait d'être arrêtée et détenue pendant 15 jours, période au terme de laquelle elle devait s'engager à trouver une occupation. Les étrangers risquaient la déportation, les récidivistes les colonies correctionnelles, voire les prisons militaires. Plusieurs personnes détenues dans ces conditions étaient envoyées à la Marine, comme en témoignent les documents du Bureau d'Identification de la Marine créé en 1908.53 En outre, le code instituait les délits de «mendicité» et d'«état d'ivresse» (articles 391 à 398), ce qui pouvait également servir les intérêts de la Marine. Notons que les individus «recrutés» par la police étaient fréquemment présentés comme des volontaires par les autorités en charge du recrutement, constituant un moyen très particulier de recrutement. Toutefois, ce recours ne fut pas le principal mode d'engagement dans le Corps de Marin Nationaux. En effet, les écoles d'apprentis marins constituaient la source de recrutement la plus fréquente, suivi par les captures et la présentation spontanée des déserteurs. Aux yeux des ministres, le nombre réduit de volontaires était dû à la suppression de la prime d'engagement et aux faibles soldes en vigueur dans la Marine, comme nous pouvons le lire dans le rapport de 1891:

Le volontariat sans une prime ne peut rien nous promettre, étant donné que, avec le récent développement de nos industries, le travail est devenu si facile et si bien rémunéré que personne ne se privera de s'y engager au lieu de s'engager comme marin contre une solde mesquine de $9 \$ 375$ par mois. C'est un montant que n'importe quel travailleur peut gagner en deux jours..$^{54}$

Selon le modèle prévu par la Constitution, un tirage au sort maritime militaire fut instauré en 1903 parmi les travailleurs de la Marine marchande âgés de 16 à 30 ans, à l'exception des «machinistes, pilotes et personnes jugées inaptes à ce service». ${ }^{55}$ Dans la mesure où on cherchait à recruter du personnel qualifié, la sélection parmi le personnel de la Marine marchande apparaissait alors comme la solution la plus adéquate.

Toutefois, ce dispositif ne permit pas d'obtenir les résultats escomptés. Une mobilisation du personnel dans les ports, l'organisation d'une grève quelques jours avant le tirage au sort et «le manque d'intérêt» du reste de la société mirent à mal sa mise en œuvre. ${ }^{56}$ Certains des individus tirés au sort obtinrent des procurations de libération de

52. Soares, Carlos Eugênio Libano, A negrada instituição. Rio de Janeiro, Arquivo Geral da Cidado do Rio de Janeiro, 1995.

53. Capanema P. de Almeida, Silvia, «Nous, marins, ...», cit., notamment chapitre 3.

54. $R M M, 1891$, p. 28

55. Décrets 4901 du 22 juillet 1903 et 4983 du 30 septembre 1903, cités dans le RMM, 1903, p. 43.

56. RMM, 1903. 
différents juges, d'autres ne se présentèrent pas à la Marine, d'autres encore se firent réformer par des médecins. Si la contribution pour les capitaines des ports fût fixée à un total de 2.000 hommes, correspondant à $50 \%$ du personnel souhaité pour totaliser l'ensemble du Corps des Marins Nationaux, seules 170 personnes avaient été enrôlées par tirage au sort le 30 avril $1904 .{ }^{57}$ Le nombre des marins tirés au sort demeura insignifiant l'année qui suivit et cette catégorie disparut par la suite.

Pour résoudre cette question des effectifs, il ne restait donc plus que les contingents sortis des écoles d'apprentis marins. Cependant, si ces institutions étaient déjà le plus important moyen de recrutement depuis la fin de la période impériale —en tant que compagnies d'apprentis—, elles devaient également passer par une série de réformes après le changement de régime. Ces écoles étaient, en effet, confrontées elles aussi au problème du sous-effectif. En outre, selon les rapports des différents ministres en poste, elles n'étaient pas en mesure d'offrir une bonne formation pour les marins, en raison de leurs mauvaises conditions d'installation et du manque d'enseignants.

De manière schématique, deux modèles de réforme furent avancés pour les écoles d'apprentis. Le premier suggérait la diminution de leur nombre pour un meilleur usage des ressources. ${ }^{58}$ Le second, au contraire, défendait l'augmentation du nombre d'écoles, pour une meilleure distribution dans les différents États du pays. ${ }^{59}$

Les écoles de l'Armada brésilienne: les modèles et l'expérience des marins et officiers de la République

L'Armada brésilienne se caractérisa par un important décalage dans la composition de son personnel entre les officiers, issus en grande partie de familles de militaires et notamment de militaires gradés, et les marins issus du milieu populaire, qui étaient à près de $80 \%$ noirs et métis, originaires des États du Nord et du Nord-est du Brésil. ${ }^{60}$ Ces deux univers sociaux étaient formés, en grande partie, grâce au passage dans les deux écoles de la Marine. Tandis que l'École Navale donnait accès aux postes d'officiers et figurait parmi les formations scientifiques les plus respectées du pays, l'école d'apprentis marins était fréquentée par des mineurs (dont de nombreux orphelins) ayant en commun de ne pas disposer d'autres perspectives. Les places dans la société restaient donc bien établies par ces deux univers, depuis l'entrée dans ces établissements. L'École Navale était destinée à former une forme de «noblesse d'état», dans le sens établi par Bourdieu. ${ }^{61}$ Si les élèves étaient déjà en grande partie originaires de cette «noblesse», le passage par l'école navale était nécessaire pour confirmer leur appartenance à ce milieu.

57. RMM, 1903, p. 51

58. Rapports de 1894, 1895, 1896, 1897, 1902, 1905.

59. Rapports de 1890, 1891, 1892, 1893, 1898, 1899, 1903.

60. Capanema P. de Almeida, Silvia, «Nous, marins, ...», cit.

61. Bourdieu, Pierre, Noblesse d'état: grandes écoles et esprit de corps, Paris, Ed. de Minuit, 1989 et par le même auteur, Langage et pouvoir symbolique, Seuil, Paris, 2001. 
Les barèmes des soldes établis après la proclamation de la République donnent une idée des écarts de rémunération et, partant, des possibilités d'évolution professionnelle entre le groupe des marins, issus ou non des écoles d'apprentis, et celui des officiers passés par l'École Navale. Selon un barème établi par un décret du 2 janvier 1890, le plus haut poste auquel normalement pouvait accéder un marin ne représentait, en termes de solde, qu'un peu plus du tiers des gains d'un officier débutant. Et, la solde d'un amiral représentait mille fois celle d'un mousse. ${ }^{62}$

Située sur l'île das Enxadas à Rio, l'École Navale proposait un cursus de quatre ans, accessible sur concours, ainsi que des classes préparatoires à ces concours, dont l'existence fut remise en question par plusieurs ministres de la Marine en raison de leur coût et du nombre restreint de postes d'officiers. Les ministres de la Marine insistaient alors sur l'importance des apprentissages pratiques. À partir de 1904, ils demandèrent en outre que soient différenciées clairement les formations des machinistes et des officiers commandants, les premiers devant recevoir une instruction plus technique. Suite à ces requêtes, des écoles pratiques furent créées afin de privilégier la formation de ces différents profils.

Les écoles s'inspiraient toujours du modèle des Armadas européennes —anglaise, allemande et française notamment-, jugé seul capable de faire entrer la Marine brésilienne dans le cercle restreint des Grandes Puissances. Un nouveau règlement fut ainsi établi en 1907 qui prévoyait, entre autres: la fusion des cours de l'école de la Marine et l'école de machines; la création de nouvelles disciplines, telles que la théorie et la construction des machines à vapeur et des navires; l'évaluation de la capacité intellectuelle comme seule condition pour intégrer l'école à travers un strict examen d'admission, éliminant les nominations par désignation ou filiation; la création de prix de mérite; la pratique de l'éducation physique.

Les élèves continuaient cependant à se distinguer par un statut social élevé. Outre le concours d'admission, faire partie de ce groupe requérait une bonne origine sociale. Dans ses mémoires, Gastão Penalva, ${ }^{63}$ un ancien élève de l'École Navale, affirme que plusieurs de ses collègues avaient de la famille dans la Marine. Nombreux étaient les fils d'officiers, parfois même d'amiraux et de ministres, cherchant à avoir un diplôme aussi côté que les diplômes de droit ou de médecine, ${ }^{64}$ qui sont devenus plus tard, des capitaines. Cela atteste des possibilités d'ascension dans la carrière.

Bien qu'appartenant à des réalités sociales très différentes, les deux catégories de personnel, officiers et marins, étaient très souvent réunies dans la formation pratique des navires-écoles, comme nous le démontre la photographie suivante, prise sur le navire-

62. RMM, 1890.

63. Penalva, Gastão, A Marinha do meu tempo, Rio de Janeiro, 1951.

64. Comme nous l'indique un témoignage de 1917, les familles brésiliennes aisées voulaient voir leurs enfants devenir des bacheliers en droit, en médecine ou en ingénierie. En dehors de ces domaines, seul restait la possibilité de devenir officier de Marine. Cf: Monteiro, Tobias, Funcionários e doutores, Rio de Janeiro, Livraria Francisco Alves, 1919 (2 $2^{\mathrm{eme}}$ ed.), p. 14, cité par: Carvalho, 1998. 
école Benjamin Constant, en 1906, en voyage en Europe (les marins portent vestes et chaussures, ce qui n'était pas toujours le cas dans les ports brésiliens).

FIGURE 1. A bord du navire école Benjamin Constant en voyage en Europe.

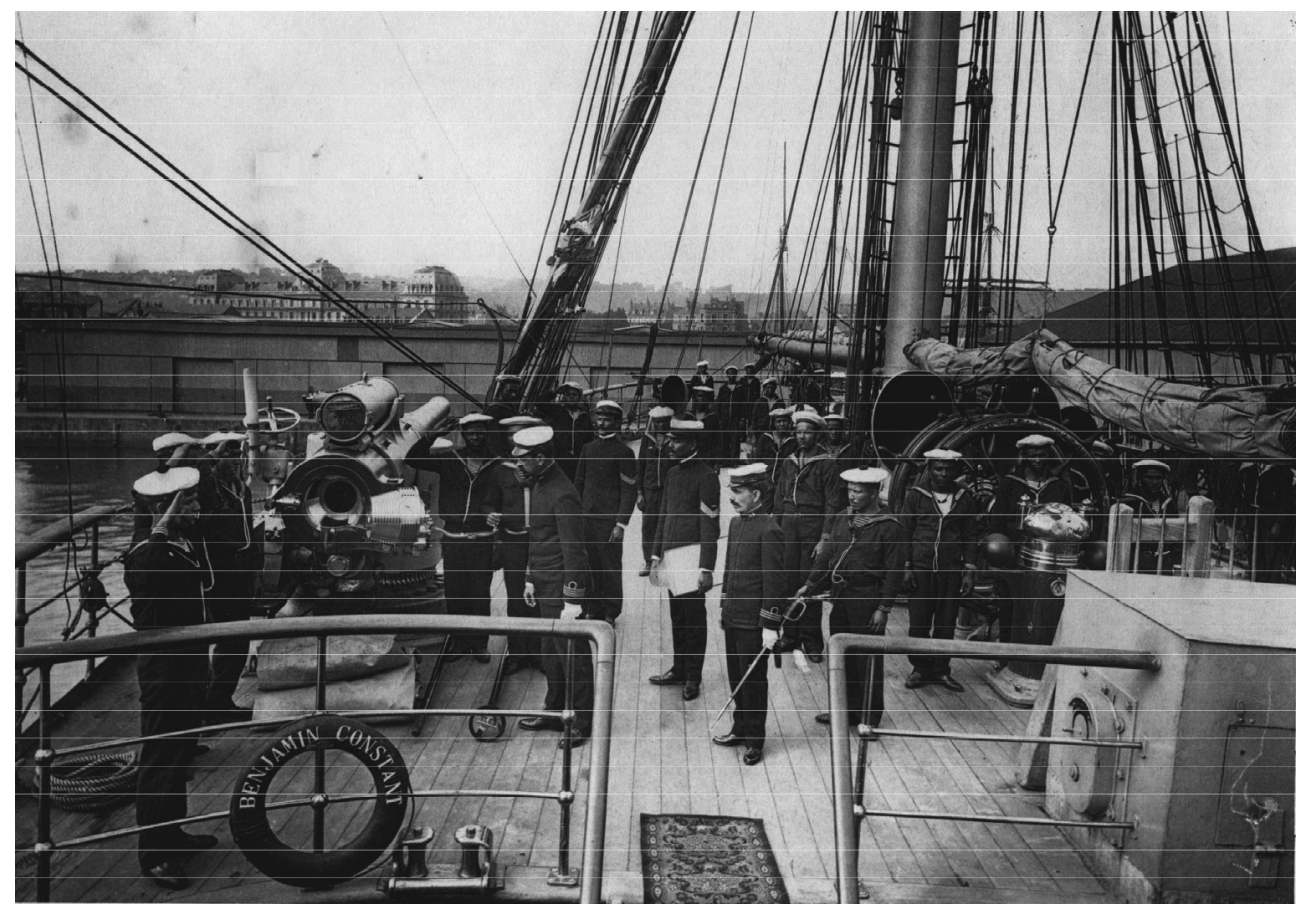

SOURCE: DPHDM-Fonds de Photos - Série Navires: Benjamin Constant, 1906.

L'image photographique (figure 1) donne à voir le salut militaire des mousses à leurs officiers, dans le navire-école, probablement dans le cadre de leur formation. Les distances sont bien marquées, ainsi que les places, et les gestes codifiés, comme en témoigne cet extrait d'un manuel de formation publié en 1908:

Les militaires de la Marine ayant les mêmes postes ou grades, lors d'une rencontre, devront se saluer réciproquement s'ils sont des officiers et, s'ils sont des sous-officiers ou marins, devront porter la main au bonnet; le compliment devant partir, dans tous les cas, du plus modeste. (...) Il devra maintenir cette position pendant qu'il parle à son supérieur et, après avoir reçu l'ordre de se retirer, fera un demi-tour, défaisant le salut et suivant son destin dans le pas qu'on lui ordonne. (.... ${ }^{65}$

65. Rocha, Francisco José Marques da (capitaine de vaisseau), Manual do Marinheiro-Fuzileiro, Rio de Janeiro, Imprensa Nacional, 1908, pp. 187-191. 
Ces règles de salut, jugées indispensables à l'établissement des rapports quotidiens dans la Marine de guerre, étaient enseignées dans les écoles. Le passage par ces institutions de formation constituait un moment d'initiation rituelle au monde militaire.

Les écoles d'apprentis étaient considérées à l'époque «le plus important vivier de la Marine de guerre» brésilienne. ${ }^{66}$ Les apprentis étaient originaires des plus basses couches de la société, souvent orphelins, conduits par des tuteurs ou des parents incapables de s'en occuper et attirés par la prime en vigueur jusqu'au début des années 1890. Cette prime pouvait atteindre $100.000 \$$, soit plus que le salaire d'un marin pendant une année de travail 96.000\$. ${ }^{67}$ Les mineurs, recrutés à des âges divers, demeuraient dans les écoles d'apprentis jusqu'à atteindre 16 ou 18 ans. Ils devenaient alors mousses dans la Marine. Ces élèves étaient censés apprendre les rudiments de la lecture et de l'écriture, les tâches de fonctionnement des bateaux et la discipline militaire.

Certains auteurs ont dénoncé le caractère colonial ou carcéral de ces écoles. ${ }^{68}$ Selon ce courant, les écoles d'apprentis marins étaient de véritables prisons dont le but n'était ni l'instruction ni la formation. Cette analyse s'appuie sur les arguments avancés à la même époque par certains ministres de la Marine, qui se défendaient de l'opinion commune: «Ancien préjugé, encore enraciné chez les autorités, selon lequel les écoles d'apprentis sont des compagnies correctionnelles pour le châtiment des mineurs pris en flagrant délit de vagabondage». ${ }^{69}$

Cette analyse prévalait également dans les milieux diplomatiques de l'époque, comme en témoigne cette note adressée par un diplomate français à son ministre de tutelle en 1910, lors de la révolte des marins dans la Baie de la Guanabara: «Les équipages, en grande majorité composés de nègres et de mulâtres, proviennent des écoles d'apprentis marins, jeunes vagabonds et jeunes malfaiteurs recueillis par la police, ou jeunes incorrigibles que leurs familles, en désespoir de cause, envoient dans ces écoles comme dans une maison de correction». ${ }^{70}$

\section{Le tournant de la période Alexandrino de Alencar}

Les réformes réalisées sous le ministère Alexandrino de Alencar (1906-1910) visaient non seulement à moderniser les équipements, mais encore à inverser cette image en restructurant les écoles d'apprentis. La tâche était loin d'être facile à en croire les nombreuses critiques émises par les officiers eux-mêmes. Nossa Marinha, une publication officielle préparée afin de diffuser et promouvoir les nouvelles mesures, en dressait un panorama

66. RMM, 1906.

67. Morgan, Zachary Ross, Legacy of the Lash: Race and Corporal Punishment in the Brazilian Navy and the Atlantic World, Indiana University Press, 2014.

68. Notamment Morgan, Zachary Ross, Legacy of ..., cit.

69. RMM, 1899.

70. Archives du Quai d'Orsay, Correspondance politique et commerciale, politique Intérieure Immigration, vol. 6, 28/11/1910. 
peu amène en 1910: «L'instruction des équipages était nulle. Les écoles se réduisaient à sept ou huit, fonctionnant dans des édifices incroyables, ayant un si faible nombre de matriculés que chaque mousse fourni à l'escadre coûtait à l'État, selon un document officiel, plus de $1.900 .000 \$ \gg .{ }^{71}$

Bien que dispendieuses pour l'État, ces écoles se trouvaient dans une situation de pénurie. C'est ce paradoxe que révélait la même publication officielle, par ce passage identifié comme étant une citation «d'un officier» au sujet de l'école de l'État de Bahia: «L'école d'apprentis marins de cet État, précisément à la même époque, était livrée à un des plus distincts officiers de la corporation. Elle comptait treize élèves! — Treize mendiants, habillés de misérables chiffons faits de sacs de patate $\gg{ }^{72}$

Mais ce nouveau projet comprenait aussi la justification de la «fonction sociale» de ces établissements, qui était celle de protéger le mineur sans famille, souvent mendiant ou délinquant, et les enfants pauvres en leur offrant un moyen de subsistance, de l'instruction et une formation professionnelle:

Le résultat de cette orientation est d'ores et déjà magnifique. Il suffit de rappeler qu'en 1908 ces viviers ont fourni à l'Armada environ 700 mousses et en 1909 rien de moins que 905, tous sachant lire et écrire, familiarisés avec la vie à bord et dressés dans une des spécialités de la Marine moderne: —signaleurs, timoniers, torpilleurs, artilleurs, miniers. ${ }^{73}$ (C'est nous qui soulignons).

Le but était donc d'instruire des mineurs et, plus que cela, de les civiliser comme l'indique la comparaison implicite entre les enfants et les animaux («vivier», «dresser»). En outre, l'investissement dans la formation était censé provoquer une diminution de la criminalité: «De cette façon, la préparation des équipages croît toujours, en même temps que —fait suffisamment notable pour être relevé— la criminalité décroît à un coefficient jamais atteint dans les effectifs militaires du pays jusqu'à maintenant, ce qui est très positif si nous le comparons aux coefficients équivalents des autres Marines». ${ }^{74}$

Ces remarques, bien que formulées dans le but de faire de la promotion des actions menées par le ministère, attestent la volonté de réformer les écoles d'apprentis (dans le discours, pour le moins). Un nouveau modèle fut alors proposé, prévoyant le partage des écoles d'apprentis entre les écoles primaires de la Marine et les «écoles modèles», ces dernières, plus précisément destinées à la formation du marin. Cette nouvelle logique devait permettre de former plus efficacement le personnel et de palier le manque chronique de bras dans la Marine.

On comptait donc 1.711 élèves en 1907, à rapporter à un total d'effectifs fixé à 5.000 marins pour la même année (ce nombre avait dans les derniers temps augmenté en raison

71. Dias, Arthur, Nossa Marinha...cit.

72. Ibidem.

73. Ibidem, p. 232.

74. Dias, Arthur, Nossa Marinha...cit., p. 264. 
de l'acquisition de vaisseaux de guerres modernes requérant plus de main d'œuvre). En termes numériques, le résultat s'avérait encourageant. Cependant, les écoles entendaient aussi privilégier l'aspect humain, la formation et la «professionnalisation» du personnel. À cet égard, il convient de noter l'optimisme qui contamina les discours des officiers et les publications officielles à partir de 1906, toujours promptes à insister sur le rôle civilisateur et philanthropique de la Marine. En témoigne cet extrait du livre Nossa Marinha (1910):

Autrefois (...) prévalait le préjugé selon lequel le service de la mer était un châtiment. Rien de plus absurde (...). Le véritable, le plus grand, le plus triste châtiment est celui qu'inconsciemment nos autorités infligent aux enfants avec l'analphabétisme et le vagabondage, qui en général les abrutissent et les rendent infortunés dans plusieurs de nos villes. Au contraire, l'élève de l'école d'apprentis reçoit sur place une formation intellectuelle, physique, morale et professionnelle qui le rend digne, le perfectionne, le sauve. ${ }^{75}$

Dans les publications officielles, ce discours était renforcé par des séries photographiques. On y voit les élèves de l'école d'apprentis marins de Rio de Janeiro dans trois situations différentes: en salle de cours, au dortoir et au réfectoire. C'est la formation, l'organisation et la propreté qui sont affichées sur ces images de promotion de la Marine comme institution qui récupère les enfants sans avenir. Nombreux sont les travaux sur l'enfance au Brésil de cette période qui signalent le grand nombre d'orphelins et d'enfants abandonnés dans les rues des villes. Enfants qui étaient parfois livrés aux institutions de l'Église, laissés sur la «roue des exposés», ${ }^{76}$ mais aussi aux institutions militaires. ${ }^{77}$

Ces images représentent toutes les trois des scènes extrêmement organisées, soulignant les bonnes conditions humaines et matérielles de l'école d'apprentis de Rio de Janeiro. La figure 2 montre des jeunes garçons allant se coucher en ordre, dans une salle parfaitement organisée et remplie de hamacs (traditionnels dans le nord-est du Brésil, mais aussi dans les Marines) et selon un rituel précis (la présence du tambour indique le signal donné pour aller se coucher). Dans une autre image, on voit plusieurs enfants en salle de cours, très concentrés, regardant un collègue qui écrit au tableau. Le maître, revêtu de l'uniforme d'officier, occupe également une place codifiée. Encore une autre photographie représente les élèves assis à table et attendant de commencer leur repas. Sur l'ensemble de ces photographies, un regard contemporain note la présence d'un grand nombre de noirs et de métis. Seuls l'officier dans le dortoir et le maître dans la salle de cours indiquent la présence d'adultes chargés de les encadrer. Les élèves ne semblent pas

75. Ibidem, p. 266.

76. Moyen d'abandonner les enfants de façon anonyme. L'enfant était placé d'un côté dans un dispositif qu'on faisait tourner pour qu'il soit reçu de l'autre côté par les institutions religieuses se trouvant dans les hospices.

77. Del Priore, Mary del (org.), Historia das crianças no Brasil, São Paulo, Contexto, 2006. 
s'être aperçus de la présence du photographe, ce qui confère aux images l'aspect documentaire d'une «réalité» quotidienne prise sur le vif. ${ }^{78}$

FIGURE 2. Ecole d'apprentis marins de Rio de Janeiro.

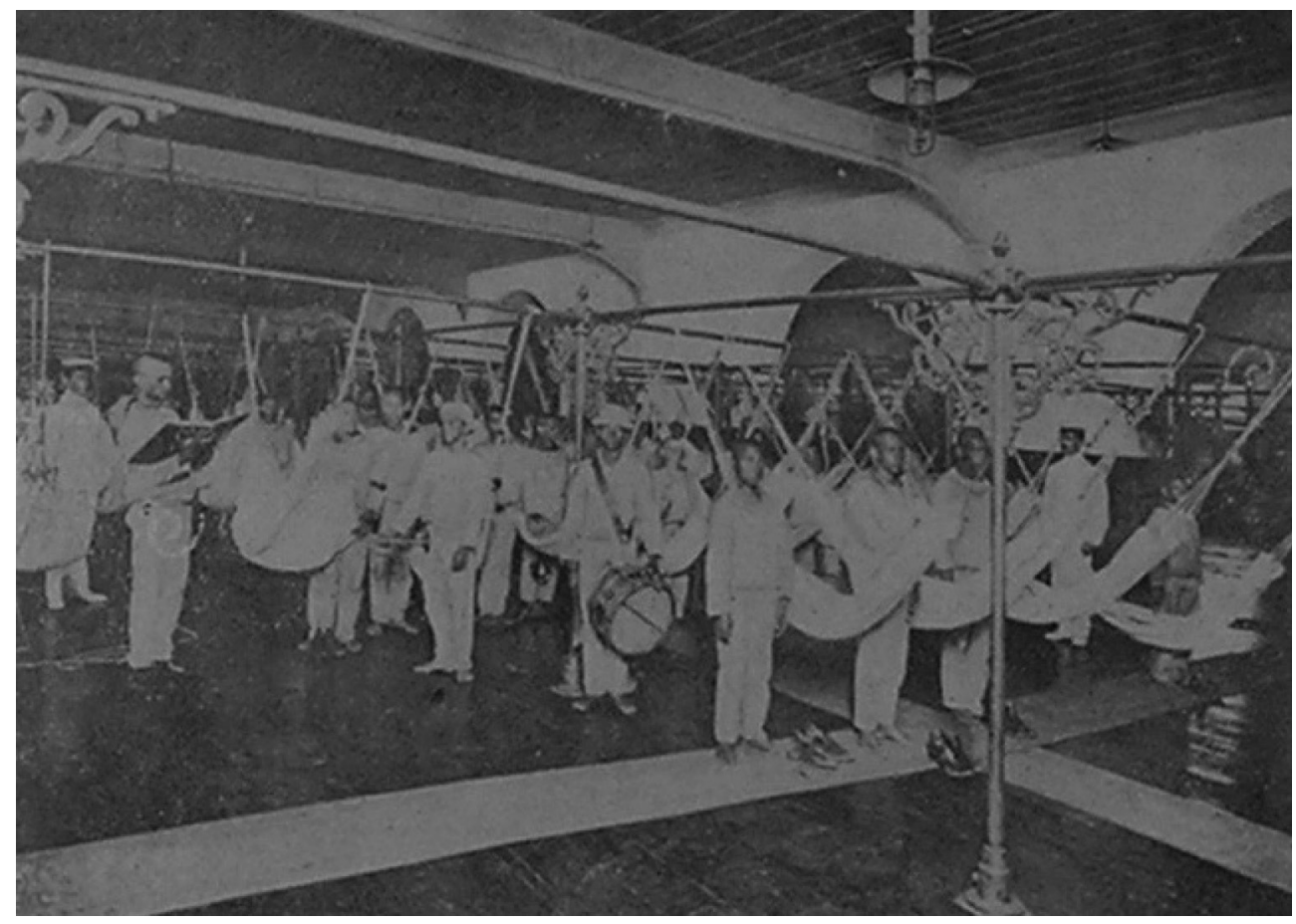

SOURCE: Dias, Arthur, Nossa Marina..., cit., 1910.

L'ancien règlement de l'école d'apprentis marins datait de 1885 et il a été remplacé par un nouveau règlement de $1907 .{ }^{79}$ En les comparant, on constate que, si les critères d'admission n'évoluèrent pas beaucoup, la prime pour l'enrôlement disparut en 1907. Le temps de service ne changea pas non plus, limité à trois ans au cours desquels l'apprenti n'avait pas beaucoup de possibilités d'arrêter l'école (les seuls moyens étant l'incapacité physique ou mentale). Selon les règlements, l'apprenti était transféré dans le Corps des Marins Nationaux à l'âge de 18 ans, et y demeurait. Toutefois, l'apprenti pouvait être transféré avant cet âge pour cause d'indiscipline - le transfert étant pris comme une punition (règlement de 1885)—, en raison de sa bonne condition physique et de la formation déjà acquise (règlement de 1885) ou, au contraire, d'une inaptitude aux études

78. Roland Barthes a bien analysé cet effet de réel de la photographie. Cf. Barthes, Roland, «La Chambre claire», Barthes, Roland. Oeuvres complètes, T. III, Paris, Seuil, 1995.

79. En annexe au RMM de 1907. 
(règlement de 1907). ${ }^{80}$ Le changement plus important entre les deux règlements concernait les contenus enseignés. Pour l'enseignement élémentaire, la lecture et l'arithmétique étaient les contenus les plus importants, mais en 1907 la doctrine chrétienne disparut et l'enseignement de la langue portugaise et de l'arithmétique se complexifia, étant mieux répartis sur les trois années d'école. Toutefois, les principales évolutions touchaient à l'enseignement professionnel et à l'introduction, dans le rapport de 1907, des enseignements optionnels. En 1907, on créa des officines pour la professionnalisation des apprentis dans d'autres corps de métiers, comme la charpenterie, la serrurerie, la quincaillerie, et l'on accorda plus de place aux activités physiques, telles que la gymnastique, la natation et même le football. En ce qui concerne les punitions, celles-ci ne subirent pas de grandes mutations, les deux règlements instituant des «punitions exemplaires» qui pouvaient inclure des châtiments corporels. En ce qui concerne le personnel encadrant, la place réservée aux enseignants extérieurs à l'Armada est marginale, la plupart des tâches, y compris l'éducation, étant à la charge des militaires.

\section{Enseignement et recrutement dans les écoles d'apprentis marin}

Dans un fichier d'inscription des mineurs dans l'école des apprentis de Bahia pendant les années 1860, on trouve quelques pistes sur le niveau d'instruction et de formation de ces jeunes apprentis, mousses ou marins. ${ }^{81}$ Pour plus d'une centaine de noms d'élèves rentrés dans la Marine entre 1859 et 1866, à quelques exceptions près, les apprentis avaient au moins des notions d'écriture et d'arithmétique, comme le montrent les registres suivants, choisis de façon aléatoire.

On remarque l'existence d'apprentis intégrés à des moments et à des âges différents (certains sont identifiés comme majeurs). Dans la plupart des cas, leur niveau d'instruction variait en fonction de leur ancienneté dans la Marine et de leur âge. Écrire bastardo ou bastardinho signifiait tracer des lettres primaires, arrondies et inclinées, donc l'écriture avant la maîtrise de la calligraphie. Écrire fino («fin») était déjà plus élaboré. Le «Bonhomme Richard», un ouvrage de Benjamin Constant, était un manuel de lecture très utilisé à cette époque au Brésil, probablement adopté dans cette école aussi. Cet ouvrage, composé de maximes qui disent comment s'enrichir et ne pas perdre son temps dans l'oisiveté, était également censé apporter une «bonne» formation morale aux jeunes, proposant toujours le chemin du travail comme opposition au vagabondage. ${ }^{82}$ Ces exemples

80. Au-delà du cadre fixé par la loi, nous constatons l'existence de plusieurs transferts avant l'âge de 18 ans, comme dans le cas du marin João Cândido, dont les raisons ne sont pas explicitées. La Marine a pu utiliser ces transferts comme un moyen de répondre au manque d'effectifs.

81. AN, Série Marinha, Fundo AP, Livro de Matriculados Menores da 2. ${ }^{a}$. Cia de Aprendizes do Arsenal de Marinha da Bahia tendo principiado a funcionar o ensino primário em 8 de setembro de 1860, Cia. De Artífices-matrícula de menores, 1860, VIII M-92.

82. Il s'agit d'une allusion à l'ouvrage de Benjamin Franklin, publié en portugais sous le titre: Franklin, Benjamin, A ciência do bom homem Ricardo, ou meios de fazer fortuna, Tipografia de Sociedade Propagadora 
d'élèves, comme tant d'autres, nous fournissent donc une idée du niveau des apprentis et de ce qu'on attendait d'eux.

Tableau 1. Les élèves de la Compagnie d'apprentis de Bahia (années 1860)

\begin{tabular}{|c|c|c|c|}
\hline $\begin{array}{l}\text { DATE D'ENTRÉE } \\
\text { DANS LA MARINE }\end{array}$ & Nom & OBSERVATIONS & STATUT \\
\hline $01 / 12 / 1865$ & Antônio de Pinho & $\begin{array}{l}\text { Lit «Bom homem Ricardo», écrit «bastardo» et } \\
\text { connaît la table d'addition }\end{array}$ & \\
\hline $02 / 02 / 1866$ & Ciro Pedrosa & $\begin{array}{l}\text { Fait le catéchisme, table de multiplication, écrit } \\
\text { «en attaché». Le } 14 \text { février a fait examen pour } \\
\text { l'histoire du Brésil. En mars, grammaire. Il a passé } \\
\text { des examens et a été approuvé. }\end{array}$ & \\
\hline $07 / 02 / 1859$ & $\begin{array}{l}\text { Horacio Augusto } \\
\text { de Matos }\end{array}$ & $\begin{array}{l}\text { Lit «Bom homem Ricardo», sait la table d'addition } \\
\text { et écrit «bastardo». En septembre } 1862 \text { a passé à } \\
\text { écrire signes. } 1863 \text { a fait le catéchisme. }\end{array}$ & Majeur \\
\hline $10 / 04 / 1863$ & $\begin{array}{l}\text { Ermelno Patricio } \\
\text { da Silva Reis Go- } \\
\text { mes }\end{array}$ & $\begin{array}{l}\text { Connaît l'abécédaire. Il a commencé le «Bom ho- } \\
\text { mem Ricardo» le } 10 \text { février } 1866 .\end{array}$ & \\
\hline $01 / 02 / 1859$ & $\begin{array}{l}\text { Leopoldo Alves de } \\
\text { Matos }\end{array}$ & $\begin{array}{l}\text { Fait le catéchisme, la grammaire, les tables de mul- } \\
\text { tiplication. A commencé à écrire des «signes» le } 10 \\
\text { février } 1861 \text {. Le } 7 \text { juillet } 1862 \text { est passé à l'histoire } \\
\text { du Brésil, à l'orthographe. Il a fait ses examens et } \\
\text { est reçu en géométrie. }\end{array}$ & \\
\hline $02 / 10 / 65$ & Lauro José Cardoso & $\begin{array}{l}\text { Fait de l'histoire du Brésil, écrit «en attaché». Il a } \\
\text { passé ses examens et a été reçu. }\end{array}$ & \\
\hline $07 / 02 / 1859$ & $\begin{array}{l}\text { Manuel Luiz de } \\
\text { Couto }\end{array}$ & $\begin{array}{l}\text { Fait le catéchisme, la grammaire portugaise, écrit } \\
\text { «fin», il a passé ses examens pour le cours de géo- } \\
\text { métrie en } 1860 \text {. }\end{array}$ & Majeur \\
\hline 08/02/1866 & $\begin{array}{l}\text { Américo Brasilio da } \\
\text { Silva }\end{array}$ & $\begin{array}{l}\text { Fait le catéchisme, connaît la table d'addition, et } \\
\text { écrit «bastardo» }\end{array}$ & \\
\hline $02 / 10 / 1865$ & $\begin{array}{l}\text { Feliciano José da } \\
\text { Silva }\end{array}$ & Lit «Bom homem Ricardo» et écrit l'abécédaire. & \\
\hline $18 / 02 / 1866$ & $\begin{array}{l}\text { José Francisco de } \\
\text {... Costa }\end{array}$ & $\begin{array}{l}\text { Fait le catéchisme, a réussi son orthographe le } 18 \\
\text { août } 1866 \text { et écrit «bastardinho» à la même date. }\end{array}$ & \\
\hline
\end{tabular}

SOurCE: AN, Série Marinha, Fundo AP, Livro de Matriculados Menores da 2. ${ }^{a}$ Cia de Aprendizes do Arsenal de Marinha da Bahia tendo principiado a funcionar o ensino primário em 8 de setembro de 1860, Cia. De Artífices- matrícula de menores, 1860, VIIIM-92.

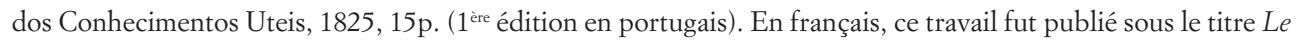
chemin de la fortune ou la science du Bonhomme Richard en 1757. Repris dans Conseils pour s'enrichir, Paris, Aléa, 1997, pp. 25-38. Au Brésil, cet ouvrage devint une référence et fut adopté comme manuel de lecture et d'instruction dans plusieurs écoles de l'Empire à la République. Voir: Almeida, João Ricardo Pires de, Historia da instrução publica no Brasil (1500-1889), São Paulo, INEP, 1989, p. 363. 
Un autre exemple est celui de l'école primaire de Paranaguá, située dans l'État du Paraná, en 1886. Le 23 décembre de cette année, le chef de division João Mendes Salgado (général adjoint de l'Armada) envoya au ministre de la Marine une lettre comprenant les résultats des élèves de son école, qu'il tenait comme très satisfaisants:

Je vous envoie ci-jointe la copie de l'acte des examens, dont le résultat a été très favorable aux crédits de cet établissement si l'on considère que, il y a peu de mois, quand l'actuel maître a commencé à enseigner, quelques apprentis étaient analphabètes. Le Dr. José Justino de Macedo et l'instituteur public Honório Décio da Costa Lobo ont été les examinateurs. Je profite de cette occasion pour vous communiquer que les vacances réglementaires commencent le 15 décembre (...). La plupart des apprentis n'ont pas de famille et très peu ont des parents qui résident ici. Ils sont toujours recueillis dans les casernes à l'heure des repas employant leur temps libre à trainer dans les rues-afin qu'ils ne restent pas trop longtemps livrés à la fainéantise, il a été recommandé que les exercices d'appareil, de manœuvre et d'aviron pour les écoliers devront avoir lieu dans les jours de la grille et que les exercices d'escrime seront journaliers, entre 9 et 10 heures, et l'infanterie de de 14 hà 15 h. ${ }^{83}$

Outre l'instruction des élèves, les écoles se devaient de les occuper, puisque la plupart n'avaient pas de famille et passaient leur temps libre à «trainer dans les rue», ce qu'il fallait absolument éviter. La lettre sous-entend également que l'organisation des écoles n'était pas toujours très structurée, l'instituteur n'ayant assumé son poste que très récemment et s'étant retrouvé face à des apprentis analphabètes. Une analyse des résultats de ces élèves donne des pistes plus précises quant à leur niveau d'instruction (bon, mauvais ou moyen): ils sont évalués quant à leur niveau en lecture de manuscrits et de la presse; calligraphie; arithmétique; grammaire portugaise; géographie du Brésil.

Quant à l'origine des élèves de l'école de Paranaguá, leurs noms de famille indiquent dans plusieurs cas une origine indigène, probablement des Guaranis ou des Indiens originaires de la branche Tupi (famille tupi-guarani) en raison des termes utilisés (Paranaguá, Ivahy, Araguary, Capivary, Guaracema, Piasseguera, Curytiba, etc.) et de leur localisation géographique. ${ }^{84} \mathrm{Il}$ convient donc de s'interroger sur le recrutement d'Indiens, qui existait certainement, mais qui a très peu été étudié par l'historiographie et qui est difficile de saisir à travers les sources. L'inscription de ces jeunes dans les écoles pour les marins serait dans une certaine mesure, une forme de ce que Darcy Ribeiro appelle de «transfiguration ethnique», ou bien une étape dans le cadre du projet civilisateur brésilien. ${ }^{85}$

L'alphabétisation et le développement de l'instruction primaire soulevèrent également de nombreux débats en Europe dans la seconde moitié du XIX ${ }^{e}$ siècle. De manière

83. Les examens datent du 10 et 11 du même mois. AN, Série Marinha, Escola de Aprendizes 1886-1888, III M702.

84. Rocha, Leandro Mendes, A politica indigenista do Brasil: 1930-1967, Goiânia, Editora UFG, 2003.

85. Ribeiro, Darcy, Os indios e a civilização: a integração das populações indigenas no Brasil moderno, São Paulo, Companhia das Letras, 1996. 
générale, la période se caractérise par un effort considérable tant du secteur privé — fréquenté par la bourgeoisie, notamment pour les écoles confessionnelles - que du secteur public — destiné aux populations plus démunies - pour instruire et socialiser les enfants. On travaillait pour le développement des connaissances fondamentales (lire, écrire, compter), mais aussi pour une plus large diffusion de la géographie et de l'histoire, des sciences naturelles, de la religion et des devoirs de citoyen et pour le développement du corps par la gymnastique. ${ }^{86}$ L'enseignement eut tendance à gagner d'autres institutions. Sabina Loriga, étudiant le passage des jeunes par les filières militaires en Europe, a montré que dans des pays comme la France, l'Armée joua un rôle important dans l'alphabétisation de la population masculine dans la seconde moitié du XIXe siècle. ${ }^{87} \mathrm{La}$ fonction éducative ne se limitait pas ici à l'alphabétisation et au calcul, mais comprenait aussi l'éducation physique et l'hygiène.

\section{L'apprenti marin: entre l'institution et l'individu}

Quelques années plus tard, une photographie montre l'école du Ceará en 1917 (figure 3). Ici est représentée la tonte des cheveux d'un apprenti marin. Cette image, emblème de la propreté et de l'organisation, édictait également l'idéal type de marin, celui qui reste loin des bagarres, des fêtes, des désordres humains et corporels de la vie de la rue, des pauvres, des orphelins et des vagabonds envoyés par la police.

Mais qui étaient ces enfants inscrits comme apprentis marins? Les nombreuses études sur les enfants abandonnés signalent, aussi bien en Europe qu'au Brésil, une importante mortalité chez les orphelins. ${ }^{88}$ Parmi ceux qui survivaient, un grand nombre servait sous les drapeaux dès l'époque moderne. Au Brésil, l'enfance abandonnée ne constitua pas cependant le principal pourvoyeur des écoles militaires. En effet, les écoles d'apprentis, construites après l'Indépendance, ne furent pas créées systématiquement dans les villes où se trouvaient les casas dos expostos (littéralement, «maison des exposés»; orphelinats). ${ }^{89}$

Outre les enfants abandonnés, les deux principales formes de recrutement des écoles d'apprentis marins étaient: l'envoi des enfants par les parents ou tuteurs et l'inscription des délinquants par la police. L'école de Bahia comptait ainsi 139 élèves en 1863, dont 102 «volontaires», 35 envoyés par la police et 2 originaires de la casa dos expostos. A l'époque où les écoles d'apprentis furent créées, tous les garçons recevaient gratuitement un trousseau et leurs parents et tuteurs touchaient une prime de cent mille réis, ce

86. Becchi, Egle et Dominique Julia, Histoire de l'enfance en occident: du XVIII siècle à nos jours, T-II. Paris, Seuil, 1998.

87. Loriga, Sabina, Soldats: un laboratoire disciplinaire. L'Armée piémontaise au XVIII siècle. Paris, Mentha, 1991, p. 36.

88. Becchi, Egle et Dominique Julia, Histoire de l'enfance..., cit.; Venâncio, Renato, «Os aprendizes da guerra», Priore, Mary del (org.). Historia das crianças no Brasil, São Paulo, Contexto, 2006.

89. Venâncio, Renato, «Os aprendizes ...», cit., p. 197. 
qui représentait alors $20 \%$ du prix d'un esclave et le coût total de deux enfants esclaves, donc une somme intéressante. Cependant, comme l'a bien montré Renato Pinto Venâncio, les parents et tuteurs n'étaient pas exclusivement motivés par des intérêts financiers: «l'analyse des documents révèle, au contraire, que l'envoi de l'enfant à la Marine pouvait signifier une attitude de préoccupation et d'attention de la part des familles, car cette institution de référence était une des rarissimes alternatives d'apprentissage professionnelle destinée à l'enfance pauvre». ${ }^{90}$

FIGURE 3. Ecole d'apprentis marins de l'état de Ceará.

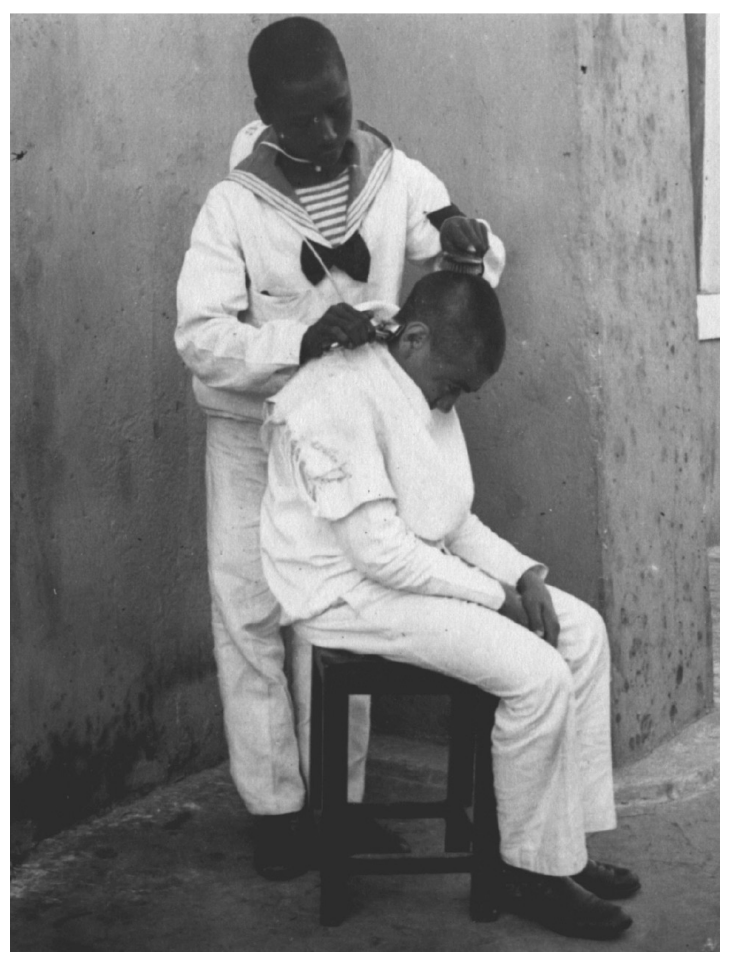

SourcE: DPHDM, Divisão de Documentos Especiais, Fundo: imagens, Série: Escola de Aprendizes: École du Ceará, 1917.

En effet, certaines familles pauvres —et les mères seules notamment_ voyaient dans les écoles de la Marine une possibilité d'ascension sociale pour leurs enfants. Pour la première fois au Brésil, «on constituait une institution véritablement publique pour les mineurs ne pouvant rester à la charge des hôpitaux ou de leurs responsables légaux».91

90. Ibidem, p. 199.

91. Venâncio, Renato, «Os aprendizes...», p. 200. 
Dans ces écoles, les mineurs étaient alphabétisés (du moins, en théorie), ce qui était appréciable dans un pays où seuls $16 \%$ des enfants de 6 à 16 ans étaient scolarisés en 1872 . Ce pourcentage eut, toutefois, tendance à augmenter dans les années qui suivirent la chute de l'Empire (1889). Les élèves disposaient aussi de journées libres (le dimanche ou le jeudi) ce qui leur permettait de maintenir le contact avec leurs familles quand celles-ci demeuraient dans la même ville.

S’inscrire à l'école d'apprentis de Porto Alegre fut la démarche que suivit le marin João Cândido, le plus important des leaders de la mutinerie de 1910. João Cândido naquit en $1880,{ }^{92}$ fils d'un père affranchi et d'une mère esclave, ${ }^{93}$ dans une ferme proche de Rio Pardo (Rio Grande do Sul) à une époque où l'institution de l'esclavage était en voie de disparition suite, notamment, à la loi du «ventre libre», en 1871 —qui libérait les enfants des esclaves quand ils avaient atteint l'âge de 21 ans. Dans un contexte aussi de l'interdiction de la traite entre les provinces en 1881, de développement du mouvement abolitionniste, avec un nombre important d'affranchissements et l'abolition de l'esclavage dans certaines provinces périphériques, dont le Rio Grande do Sul en 1884.94

Après l'abolition, la famille de João Cândido quitta la campagne pour s'installer successivement à Rio Pardo et à Porto Alegre, où ils se placèrent sous la protection de Alexandrino de Alencar, futur ministre de la Marine, également originaire de Rio Pardo. João Cândido serait donc entré dans la Marine par le biais d'Alexandrino de Alencar, qui espérait ainsi offrir un avenir à l'enfant de l'ancien troupier João Felisberto et d'Ignácia, qui était aussi sage-femme selon Morel. ${ }^{55}$ Selon un autre auteur, João Cândido allait souvent chez Alexandrino de Alencar, qui avait un grand pavillon à Rio Pardo, de nos jours le musée de la ville: «Avec la bénédiction de l'amiral Alexandrino de Alencar, João Cândido a pu se projeter dans la vie. Adressé à la Marine, qui exerçait à l'époque le châtiment corporel pour les gens déréglés et rebelles, il a révélé à la société l'homme bon qui existait dans le garçon noir». ${ }^{96}$

Avant d'entrer dans la Marine, cependant, João Cândido avait déjà intégré l'Armée de Terre en 1893, «obligé par des raisons de force majeure» comme il l'expliqua quelques années plus tard, dans les colonnes de la Gazeta de Notícias du 31 décembre 1912. Il aurait ensuite été transféré à l'école d'apprentis marins du Rio Grande do Sul en décembre 1894 ou en janvier 1895. C'est à ce moment précis de son histoire que commence le pre-

92. Deux dates de naissance furent enregistrées: le 24 juin, date reconnue par le marin lui-même, et le 15 janvier, selon son extrait de baptême étudié par Nascimento (2011). A l'époque, les dates de naissance n'étaient pas toujours connues. Il est probable que João Cândido ait choisi la date du 24 juin comme sa date de naissance puisqu'il s'agit de la Saint Jean (São João, en portugais). Cf. Nascimento, Alvaro Pereira do, Um jovem negro no pos abolição: do Ventre livre à Marinha de Guerra, Carvalho, José Murilo e Adriana Pereira Campos (orgs.), Perspectivas da cidadania no Brasil Império, Rio de Janeiro, Civilização Brasileira, 2011.

93. Ibidem.

94. Mattos, Hebe, Das cores do silêncio: o significado da liberdade no sudeste escravista, Rio de Janeiro, Nova Fronteira, 1998.

95. Morel, Edmar, A revolta da chibata, Rio de Janeiro, Paz e Terra, 2009.

96. Ferreira, Maria Luci Correa, Tributo a João Cândido: o rei do farol da liberdade, Encruzilhada do Sul, 2002, p. 42. 
mier chapitre de son autobiographie, publiée sous le titre «Mémoires de João Cândido, le Marin» dans la Gazeta de Notícias en $1912 .^{97}$

Il fréquenta cet établissement pendant 11 mois, avant d'être incorporé dans la Marine en tant que mousse dans la capitale fédérale (sans doute pour combler les pertes d'effectifs consécutives à la révolte de l'Armada). ${ }^{98}$ Les contingents de la Marine ne comptaient, en effet, que 1.708 hommes en 1895 pour un total initialement fixé à 4.000. À l'inverse, l'école du Rio Grande do Sul, qui comptait 92 apprentis pour 100 pressentis, présentait un taux de remplissage rare pour l'époque.9

Il fut donc inscrit dans le Corps des Marins Nationaux entre 14 et 15 ans, contrairement au règlement de l'époque, qui n'autorisait théoriquement que l'inscription des individus âgés de plus de 18 ans à de rares exceptions près. De l'école d'apprentis du Sud aux bateaux de la Marine à Rio, il avait appris à lire, à broder et à maîtriser les techniques de navigation et de guerre.

De ses souvenirs d'école, il parla peu, se bornant à reconnaître l'importance de la Marine dans sa formation personnelle: «Je suis rentré à la Marine à 14 ans et j'y suis rentré novice (...). Toute la lumière qui m'a illuminée, qui m’illumine toujours, grâce à Dieu, ce peu de lumière qui n'est pas beaucoup, a été acquise, je peux le dire, dans la Marine». ${ }^{100}$ João Cândido était lettré, ou du moins il savait lire, comme en témoignent différentes photographies où il apparait et les déclarations qu'il faisait souvent à la presse. ${ }^{101}$

Le cas de Miníbio Pereira da Silva, marin passé devant le tribunal militaire de 1912 pour avoir fait partie du soulèvement de 1910, atteste également le rôle joué par les écoles d'apprentis. Parmi les pièces jointes au procès, nous avons trouvé une lettre de sa mère, qui évoque l'école des apprentis à la fois comme une opportunité de promotion sociale et comme un lieu de mauvaises fréquentations, relevant ainsi toute l'ambiguïté de ces établissements:

Mon cher Miníbio, c'est avec le cœur bien triste que je prends cette plume afin de te rencontrer mieux, mon fils. (...) Tes amis traîtres. Ne te souviens-tu pas, Miníbio, combien tu as souffert dans cette école à cause des plus grands... Tu t'en es allé et tu es parti en Europe, tu étais si heureux (...) ton casier était blanchi. Comment as-tu pu changer ta pensée et suivre le chemin pris par tes amis? (...) Oublie-les, ne te rappelle pas d'eux et ne cherche pas à parler avec ces traîtres. Obéis-moi... ${ }^{102}$

97. «Memórias de João Cândido, o marinheiro», Gazeta de Notícias, 31/12/1912.

98. Morel, Edmar, A revolta da..., cit.

99. Selon RMM, 1895, p. 24.

100. Mis, João Cândido, o almirante negro, Rio de Janeiro, Gryphus/ Museu da Imagem e do Som, 1999, p. 87.

101. Capanema P. de Almeida, Silvia, «Nous, marins, ...», cit.

102. AN, Supremo Tribunal Militar, Processo «João Cândido e outros», Série Judiciária, Subsérie: Processo Crime; 1913, BW 2847, V. 1, f. 18. 
La figure de l'enfance vagabonde était déjà une réalité fréquente dans les grandes villes du Brésil au tournant des XIXe et XX ${ }^{e}$ siècles. Une étude montre qu'entre 1900 et 1916, le coefficient des prisons pour 10.000 habitants était de 307,32 majeurs et 275,14 mineurs. Parmi ceux-ci, entre 1904 et 1906, $40 \%$ avaient été emprisonnés pour «désordres», $20 \%$ pour «vagabondage», $17 \%$ pour « état d'ivresse», $16 \%$ pour « vol » et le reste pour d'autres crimes, dont homicides. ${ }^{103}$ Le rapport du ministre de la Marine de 1891 atteste le lien établi entre enfants abandonnés, vagabondage et écoles d'apprentis:

Aucune des écoles existantes n'a le nombre complet d'apprentis et ce fait est dû à la répugnance des parents et des tuteurs qui ne veulent pas destiner leurs enfants à cette rude et aventureuse profession; et au mépris de certaines autorités, qui pourraient et devraient aider à l'enrôlement; et encore à la suppression de la prime concédée auparavant. (...) Cependant, des centaines d'enfants désemparés, qui trouveraient dans ces écoles, outre l'hébergement, le pain du corps et de l'esprit, et deviendraient ainsi des citoyens utiles à eux-mêmes et à la Patrie, vaguent fainéants par les rues et les places des villes et villages, dans tous les États, acquérant tout genre de vices et, ainsi, devenant les criminels de l'avenir, quand il ne leur arrive pas de mourir en raison de toutes ces misères! $!^{104}$

\section{Conclusion}

L'aboutissement du projet d'acquisition de nouveaux bâtiments de guerre fait partie d'un processus complexe lié au désir de moderniser la Marine. Les modèles étrangers comptaient beaucoup, fournissant autant de justifications pour le choix de tel ou tel projet, selon le regard et la conception des différents ministres de la Marine. Si ces choix furent en grande partie politiques, ils furent également influencés par les rapports que les différents ministres nourrissaient avec les chantiers des grandes puissances. À l'étranger, les projets navals brésiliens étaient donc vus tantôt comme une opportunité de bonnes affaires, tantôt comme une menace —notamment en ce qui concerne les rapports que les États européens avaient entre eux ou bien l'équilibre des forces en Amérique du Sud. À l'intérieur du pays, l'arrivée de ces bâtiments, présentés comme de véritables symboles de la République, suscita l'enthousiasme populaire, même si des critiques ne tardèrent pas à se faire entendre.

De fait, si le besoin de moderniser la Marine était reconnu de manière consensuelle, les façons d'y parvenir ne faisaient pas l'unanimité parmi les différents acteurs du jeu politique et militaire de l'époque, qui s'opposèrent avec la même verve sur le projet de restructuration du personnel de la Marine. La Marine de la fin de l'Empire et du début de la République doit faire face à un problème constant de sous-effectifs. Les désertions

103. Santos, Jocélio Teles dos, « De pardos disfarçados a brancos claros: classificações raciais no Brasil do século XVIII-XIX», Afro-Asia, n. 35, 2005, pp. 115-137, 214.

104. RMM, 1891, p. 32. 
étaient nombreuses et les formes de recrutements inefficaces. Les conditions de travail étaient très dures, avec de basses soldes, déjà pas compétitives pour le marché de l'époque, et de longues périodes de service obligatoire, une discipline rigide avec la permanence des châtiments corporels. Le recrutement par la police à travers les dispositifs existant dans le code pénal républicain de 1890 (le «vagabondage», la «mendicité» et la «pratique de capoeira») fonctionnait comme un repoussoir et provoquait des tensions supplémentaires entre officiers et marins. Le tirage au sort n'étant pas respecté, il ne restait que l'important «vivier» des écoles d'apprentis. Encouragées car pouvant résoudre à la fois le «problème» de l'enfance errante et du besoin de formation des marins subalternes pour une Marine de guerre moderne, elles seront la principale solution pour résoudre le manque de personnel dès la fin du XIX ${ }^{\mathrm{e}}$ siècle.

Le projet de modernisation de la Marine comprenait en fait deux aspects: le renouvellement du matériel d'une part, le recrutement et la formation du personnel d'autre part. Mais ces deux projets étaient incomplets et comprenaient autant de limites que de contradictions. Or, c'est précisément cette modernisation inachevée et les préjugés dont elle était porteuse qui furent questionnés par l'éclatement de la révolte des marins de 1910 contre les châtiments corporels et pour le respect de la citoyenneté, une promesse républicaine, à partir des bases acquises malgré tout dans les transformations proposées et mises en place par la Marine du tournant du XIX vers le XX ${ }^{\mathrm{e}}$ siècle.

\section{Sources citées}

AN (Archives Nationales de Rio de Janeiro), Série Marinha.

Brasil, História Naval Brasileira, Rio de Janeiro, Ministério da Marinha-Serviço de Documentação da Marinha, 1997.

DPHDM (Diretoria do Patrimônio Histórico e Documentação da Marinha), Divisão de Documentos Especiais, Fundo: imagens, Série: Escola de Aprendizes.

Rapports du Ministre de la Marine (RMM), 1888-1911. Disponible sur: http://ddsnext.crl.edu/title s?f $\% 5$ B0 \% 5D=collection $\% 3$ ABrazilian $\% 20$ Government $\% 20$ Documents\&f $\% 5 \mathrm{~B} 1 \% 5 \mathrm{D}=$ grou ping \%3AMinisterial\%20Reports.

O Paiz, 18/04/1910 (périodique de tendance conservatrice de circulation quotidienne, Rio de Janeiro, 1884-1930).

\section{Bibliographie}

Abreu, Martha. (2010). O «crioulo Dudu»: participação política e identidade negra nas histórias de um músico cantor (1890-1920). Topoi (Rio de Janeiro), 11 (20), 92-113. ALENCAR, Carlos Ramos de, Alexandrino, o grande marinheiro, Rio de Janeiro, Serviço de Documentação Geral da Marinha, 1989.

Almeida, João Ricardo Pires de, Historia da instrução publica no Brasil (1500-1889), São Paulo, INEP, 1989. 
Alonso, Angela, Flores, votos e balas: O movimento abolicionista brasileiro (1868-88), São Paulo, Companhia das Letras, 2015.

Arendt, Hannah, Les origines du totalitarisme: l'impéralisme (1951), Paris, Fayard, 1982.

Arias Neto, José Miguel, Em busca da cidadania: praças da Armada nacional, 1867-1910; Thèse de doctorat, Departamento de Historia, Faculdade de Filosofia, Letras e Ciências Humanas, Universidade de São Paulo, 2001.

Barbosa, Rui, «Lição do Extremo Oriente», Cartas da Inglaterra [1895], São Paulo, Iracema, 1972, pp. 11-92.

Barthes, Roland, «La Chambre claire», Barthes, Roland. Oeuvres complètes, T. III, Paris, Seuil, 1995.

Beattie, Peter, The Tribute of Blood: Army, Race and Nation in Brazil, 1864-1945, Durham, Duke University Press, 2001.

Becchi, Egle e Dominique Julia, Histoire de l'enfance en occident: du XVIII siècle à nos jours, T-II. Paris, Seuil, 1998.

Bourdieu, Pierre, Noblesse d'état: grandes écoles et esprit de corps, Paris, Ed. de Minuit, 1989.

-, Langage et pouvoir symbolique, Seuil, Paris, 2001.

Bueno, Clodoaldo, Politica externa da Primeira Republica: os anos de apogeu —de 1902 a 1908, São Paulo, Paz e Terra, 2003.

Caminha, Herick Marques, Historia administrativa do Brasil: organização administrativa do Ministério da Marinha na Republica, Brasilia/Rio de Janeiro, Serviço de Documentação da Marinha, 1989.

Capanema, P. de Almeida, Silvia, 'Nous, marins, citoyens brésiliens et républicains': identités, modernité et mémoire de la révolte des matelots de 1910, Thèse de doctorat en histoire, EHESS, Paris, décembre 2009, dirigé par Juan Carlos Garavaglia.

Carvalho, José Murilo de, Pontos e bordados: escritos de historia e politica, Belo Horizonte, Ed. UFMG, 1998.

Costa, Emilia Viotti da, Da Monarquia à Republica: momentos decisivos, São Paulo, Grijalbo, 1977.

Del Priore, Mary del (org.), Historia das crianças no Brasil, São Paulo, Contexto, 2006.

Dias, Arthur, Nossa Marinha: notas sobre o renascimento da Marinha de guerra do Brazil no quatriennio de 1906 a 1910, Rio de Janeiro, Officinas Graphicas da Liga Maritima Brazileira, 1910.

Duby, Georges (dir.), Grand atlas historique, Paris, éditions Larousse, 2006.

Ferreira, Maria Luci Correa, Tributo a João Cândido: o rei do farol da liberdade, Encruzilhada do Sul, 2002.

Franklin, Benjamin, A ciência do bom bomem Ricardo, os meios de fazer fortuna, Tipograria de Sociedade Propagadora dos Conhecimentos Uteis, 1825 ( 1 ère édition en portugais). [en français, Le chemin de la fortune ou la Science du Bonhomme Richard, 1757]

Greenhalgh, Juvenal, O arsenal de Marinha do Rio de Janeiro na bistória: 1822-1889, Rio de Janeiro, IBGE, 1965.

Heinsfeld, Adelar, «Estanislau Severo Zeballos: artifice da politica armamentista argentina no inicio do século XX», communication présentée dans la 26a. Réunion de la Sociedade Brasileira de Pesquisa Historica, julho 2006.

Lenhof, Jean-Louis, «Voile ou vapeur: le travail et la vie à bord des cargos français à la fin du XIX siècle (1880-1920)», Revue d'Histoire Maritime, n. 5, 2006.

Loriga, Sabina, Soldats: un laboratoire disciplinaire. L'Armée piémontaise au XVIII siècle. Paris, Mentha, 1991. 
Martins Filho, João Roberto, A Marinha brasileira na era dos encouraçados, 1885-1910, Rio de Janeiro, editora FGV, 2010.

Mattos, Hebe, Das cores do silêncio: o significado da liberdade no sudeste escravista, Rio de Janeiro, Nova Fronteira, 1998.

Mattos, Ilmar Rohloff de, «Construtores e herdeiros: a trama dos interesses na construção da unidade politica», Almanack Braziliense, n. 01, mai 2005.

Mis, João Cândido, o almirante negro, Rio de Janeiro, Gryphus/ Museu da Imagem e do Som, 1999.

Monteiro, Tobias, Funcionários e doutores, Rio de Janeiro, Livraria Francisco Alves, 1919 (2 $2^{\text {ème }}$ ed.).

Morel, Edmar, A revolta da chibata, Rio de Janeiro, Paz e Terra, 2009.

Morel, Marco, A transformação dos espaços publicos: imprensa, atores politicos e sociabilidades na cidade imperial (1820-1840), São Paulo, Hucitec, 2005.

Morgan, Zachary Ross, Legacy of the Lash: Race and Corporal Punishment in the Brazilian Navy and the Atlantic World, Indiana University Press, 2014.

Moura, Cristina Patriota de, «Herança e metamorfose: a construção social de dois Rios Brancos», Estudos Historicos, n. 25, 2000/1, pp. 81-101.

Nascimento, Alvaro Pereira do, Um jovem negro no pos abolição: do Ventre livre à Marinha de Guerra, Carvalho, José Murilo e Adriana Pereira Campos (orgs.). Perspectivas da cidadania no Brasil Império, Rio de Janeiro, Civilização Brasileira, 2011.

Neves, Lucia Maria de Bastos das e Humberto Fernandes Machado, O Império do Brasil, Rio de Janeiro, Nova Fronteira, 1999.

Penalva, Gastão, A Marinha do meu tempo, Rio de Janeiro, 1951.

Ribeiro, Darcy, Os indios e a civilização: a integração das populações indigenas no Brasil moderno, São Paulo, Companhia das Letras, 1996.

Rocha, Francisco José Marques da (capitaine de vaisseau), Manual do Marinheiro-Fuzileiro, Rio de Janeiro, Imprensa Nacional, 1908.

Rocha, Leandro Mendes, A politica indigenista do Brasil: 1930-1967, Goiânia, Editora UFG, 2003.

Santos, Jocélio Teles dos, «De pardos disfarçados a brancos claros: classificações raciais no Brasil do século XVIII-XIX», Afro-Asia, n. 35, 2005, pp. 115-137.

Scarvada, Levy, Historia do corpo de suboficiais da Armada, Imprensa Naval, Rio de Janeiro, 1954.

Schwarcz, Lilia Moritz, O espetaculo das raças: cientistas, instituições e questão racial no Brasil (1870-1930), São Paulo, Cia das Letras, 2005.

Soares, Carlos Eugênio Libano, A negrada instituição. Rio de Janeiro, Arquivo Geral da Cidado do Rio de Janeiro, 1995.

Taillemite, Etienne, L'Histoire ignorée de la Marine française, Paris, Perrin, 1999.

Venâncio, Renato, «Os aprendizes da guerra», Priore, Mary del (org.). Historia das crianças no Brasil, São Paulo, Contexto, 2006.

Verge-Franceschi, Michel, Dictionnaire d'histoire maritime. Paris: Editions Robert Laffont, 2002. 